TITLE:

\title{
A mutation of the fission yeast EB1 overcomes negative regulation by phosphorylation and stabilizes microtubules.
}

\section{$\operatorname{AUTHOR}(\mathrm{S})$ :}

limori, Makoto; Ozaki, Kanako; Chikashige, Yuji; Habu, Toshiyuki; Hiraoka, Yasushi; Maki, Takahisa; Hayashi, Ikuko; Obuse, Chikashi; Matsumoto, Tomohiro

\section{CITATION:}

limori, Makoto ...[et al]. A mutation of the fission yeast EB1 overcomes negative regulation by phosphorylation and stabilizes microtubules.. Experimental cell research 2012, 318(3): 262-275

\section{ISSUE DATE:}

2012-02-01

URL:

http://hdl.handle.net/2433/153281

\section{RIGHT:}

(c) 2011 Elsevier Inc.; この論文は出版社版でありません。引用の際には 出版社版をご確認ご利用ください。; This is not the published version. Please cite only the published version. 


\section{A mutation of the fission yeast EB1 overcomes negative regulation by phosphorylation and stabilizes microtubules.}

Makoto Iimori ${ }^{1}$, Kanako Ozaki ${ }^{1}$, Yuji Chikashige ${ }^{2}$, Toshiyuki Habu ${ }^{1,3}$, Yasushi Hiraoka $^{2,4}$, Takahisa Maki ${ }^{5}$, Ikuko Hayashi ${ }^{5}$, Chikashi Obuse ${ }^{6}$ and Tomohiro Matsumoto *1,3

1: Graduate school of Biostudies, Kyoto University, Kitashirakawa-Oiwake cho, Sakyo ku, Kyoto, 606-8502, Japan

2: Kobe Advanced ICT Research Center, National Institute of Information and Communications Technology, Kobe, 651-2492, Japan

3: Radiation Biology Center, Kyoto University, Yoshida-Konoe cho, Sakyo ku, Kyoto, 606-8501, Japan

4: Graduate School of Frontier Biosciences, Osaka University, 1-3 Yamadaoka, Suita, Japan 565-0871

5: Graduate School of Nanobioscience, Yokohama City University

Tsurumi, Yokohama, 230-0045, Japan

6: Graduate School of Life Science, Hokkaido University, Sapporo 001-0021, Japan

Running title: Regulation of fission yeast EB1

Corresponding author: Tomohiro Matsumoto

Radiation Biology Center, Kyoto University, Yoshida-Konoe cho, Sakyo ku, Kyoto, 606-8501, Japan

Phone: 81-75-753-7552

Fax: 81-75-753-7564

Mail: tmatsumo@house.rbc.kyoto-u.ac.jp 
Summary: Mal3 is a fission yeast homolog of EB1, a plus-end tracking protein (+TIP). We have generated a mutation (89R) replacing glutamine with arginine in the calponin homology $(\mathrm{CH})$ domain of Mal3. Analysis of the 89R mutant in vitro has revealed that the mutation confers a higher affinity to microtubules and enhances the intrinsic activity to promote the microtubule-assembly. The mutant Mal3 is no longer a +TIP, but binds strongly the microtubule lattice. Live cell imaging has revealed that while the wild type Mal3 proteins dissociate from the tip of the growing microtubules before the onset of shrinkage, the mutant Mal3 proteins persist on microtubules and reduces a rate of shrinkage after a longer pausing period. Consequently, the mutant Mal3 proteins cause abnormal elongation of microtubules composing the spindle and aster. Mal3 is phosphorylated at a cluster of serine/threonine residues in the linker connecting the CH and EB1-like C-terminal motif domains. The phosphorylation occurs in a microtubule-dependent manner and reduces the affinity of Mal3 to microtubules. We propose that because the $89 \mathrm{R}$ mutation is resistant to the effect of phosphorylation, it can associate persistently with microtubules and confers a stronger stability of microtubules likely by reinforcing the cylindrical structure. 


\section{Introduction}

Microtubules are highly dynamic polymers that constantly switch between phases of growth and shrinkage [1]. Microtubule dynamics at the plus end, called dynamic instability, is important for proper cellular functions, such as cell division, differentiation and migration [2-4]. Plus-end tracking proteins (+TIPs), which accumulate selectively at growing microtubule plus ends [5-7] are considered to play an important role in regulation of stability of microtubules. EB1, originally identified as a binding partner of the adenomatous polyposis coli (APC) tumor suppressor [8], is one of the +TIPs highly conserved among eukaryotes. The subsequent studies demonstrated that most of the other +TIPs that can directly interact with microtubules are also able to bind to EB1, suggesting that EB1 may function as a central hub in the network of +TIPs [9]. EB1 associates with the microtubule filament as a dimer [10, 11] via the calponin homology $(\mathrm{CH})$ domain at the amino-terminal $[12,13]$. EB1 and Mal3, a fission yeast homolog of EB1, can promote microtubule assembly in vitro [14-16]. The effects of these proteins on microtubule dynamics vary between the experimental systems. While they stimulate growth initiation and suppress catastrophes in vivo $[10,17]$, they stimulate both catastrophe and rescue in reconstituted systems $[10,16,18]$. Recent studies have also shown that Mal3 decorate the microtubule lattice seams $[15,16,19]$, suggesting that binding of EB1/Mal3 stabilizes the cylindrical structure of the microtubule.

As deletion of $\mathrm{mal3}^{+}$gene does not lead to the cell lethality, it provides an opportunity to study the effect of loss of function of Mal3 on a number of biological processes in fission yeast. Loss of Mal3 leads to abnormal short microtubules [20]. Mal3 recruits the kinesin Tea2 onto the cytoplasmic microtubules, and promotes Tea2 motor activity [21]. Tea2 transports Tip1, the fission yeast homolog of CLIP-170 [22], 
toward the growing microtubule plus ends in Mal3-dependent manner [17, 18, 23].

Like EB1 in higher eukaryotes, Mal3 also plays an important role at the kinetochore in fission yeast. It binds Spc7, a member of the conserved Spc105/KNL-1 family of kinetochore proteins, required for the integrity of the spindle as well as for targeting of MIND complex to the kinetochore [24, 25]. Deletion of $\mathrm{mal3}^{+}$gene results in a delay in mitosis, which is dependent on the Bub1-dependent spindle checkpoint. As simultaneous deletion of $\mathrm{mal3}^{+}$and $\mathrm{bubl}^{+}$genes causes monopolar attachment of sister centromeres, Mal3 cooperates with Bub1-dependent spindle checkpoint to promote bipolar attachment [26].

The activity of EB1/Mal3 to stabilize microtubules and promote its assembly must be regulated in vivo. We attempted to generate a mal3 mutant, which could deviate from regulation. Here we report characterization of such a mutant both in vivo and in vitro. 


\section{Results}

\section{Construction of mal3-89R mutant}

We aimed to investigate a mechanism to regulate Mal3 and the consequence of a failure in such regulation. A pool of the mal3 mutants was generated by error-prone PCR and transformed into a wild type fission yeast strain after cloning into pREP81. Among approximately $1.6 \times 10^{5}$ transformants screened, we obtained a plasmid that caused a growth defect upon induction of the gene hereafter designated mal3-89R. (Fig.S1A). The growth defect caused by overexpression of the mal3-89R gene was largely due to a delay in mitosis Judged by chromosome condensation as a mitotic index (MI), $22.3 \%$ of cells were in mitosis when the mal3-89R was overexpressed. The wild type $\mathrm{mal3}^{+}$gene did not affect growth (Fig.S1A) or cause a delay in mitosis (MI: $2.0 \%$ ) when overexpressed from pREP81. Overexpression of mal3-89R in mutants defective in the spindle checkpoint function caused a much severer growth defect (Fig. S1C), indicating that it activated the checkpoint.

The mal3-89R mutant contained a single point mutation replacing glutamine at the position 89 with arginine (Q89R) within the $\mathrm{CH}$ domain important for microtubulebinding $[12,13]$ (Fig. 1A). As shown in Fig. 1B, the mutation of Q89R was predicted to locate on the putative microtubule-binding surface in the $\mathrm{CH}$ domains.

Replacement of a polar amino acid to a basic amino acid would affect electrostatic interaction with microtubules and might alter microtubule dynamics. To test this possibility, we examined the mutant protein for its effect on a microtubule assembly assay in vitro. Tubulin was purified from bovine brain and a polymerization assay was performed at $37^{\circ} \mathrm{C}$ in the presence of the wild type or mutant Mal3 proteins purified 
from bacteria. The mutant protein promoted assembly more strongly than the wildtype proteins (Fig. 1C).

\section{Phenotypes of mal3-89R; abnormal morphology/dynamics of microtubules}

To examine the effects of the mal3-89R mutation under physiological conditions, we replaced the $\mathrm{mal3}^{+}$gene with the mal3-89R in a haploid strain. The resulting mal3$89 R$ mutant, that is identical to wild type strains except for the presence of the mutation, could grow at a rate comparable to that of wild type strains at any temperature we tested $\left(20,26,32\right.$ and $\left.37^{\circ} \mathrm{C}\right)$. Although overexpression of mal3-89R caused a delay/arrest imposed by the spindle checkpoint, expression of mal3-89R form the native promoter did not do so. In a genetic background defective in the spindle checkpoint, the mal3-89R mutant did not exhibit any noticeable phenotypes. We also examined the sensitivity to a microtubule poison, TBZ (Thiabendazole) and found that the mal3-89R mutant was as sensitive as the wild type strain to the drug (Fig. S2). The mal3-89R mutation thus cannot confer resistance to the drug that is proposed to target the $\alpha$-tubulin monomer [27]. Closer examination, however, revealed a number of defects in events involving microtubule dynamics. In the wild type fission yeast cells, cytoplasmic microtubules disappear around the onset of mitosis and are replaced with the spindle microtubules associated by short astral microtubules at the minus ends. We confirmed this mitotic configuration of microtubules in the wild type cells expressing $\alpha$-tubulin tagged with GFP [28] (Fig. 2A). Remarkably, in the mal3-89R mutant, we noticed robust astral microtubules, which often reached the cell tip and further extended along the cell cortex. The mitotic spindle was also abnormally extended and bent between the two poles. In addition, disassembly of cytoplasmic microtubules was occasionally incomplete in the 
mitotic cells (Fig. 2A and B). A similar phenotype was observed in klp5 and klp6 mutants [29]. In interphase, the cytoplasmic microtubules were morphologically normal (Fig. 2A). These phenotypes were not artifacts caused by the GFP-tagging as abnormal morphology of microtubules in the mutant was confirmed by indirect immunofluorescent staining with an antibody to $\alpha$-tubulin (Fig. 2C).

We also monitored progression through mitosis after the cdc25-arrest. A cdc25 mutant and a double mutant, $c d c 25-22$ mal3-89R, both of which were expressing GFP-tagged Sid4 as a marker for SPB $[30,31]$ were arrested at the G2/M boundary and released into the permissive temperature $26^{\circ} \mathrm{C}$. As shown in Fig. S3A, progression of mitosis was delayed for approximately 30 minutes in the $c d c 25-22$ mal3-89R double mutant. The delay was also apparent in analysis of the distance between the two poles. By 30 minutes after the release, the SPBs did not separate in most of the double mutants (Fig. S3B), suggesting that the delay in progression through mitosis was largely attributable to the delay in separation of SPBs at the onset of mitosis. As shown in Fig. S1B-j, overexpression of mal3-89R form pREP81 resulted in a mitotic arrest with monopolar spindle, suggesting that Mal3-89R inhibited an initial step of the SPB separation (such as interdigitation of the mitotic spindle). Even when expressed from the native promoter, it might cause a transient delay in the SPB separation. Observation by a time-lapse microscope indicated that progression after the onset of mitosis was delayed modestly in the mal3-89R mutant (Fig. S3C and D). We also monitored the stability of an artificial chromosome Ch16 [32] and found it was lower in the mal3-89R mutant and $\Delta$ mal3 (Table 1). The stability was particularly low at $36^{\circ} \mathrm{C}$. Although speculative, an important process for faithful segregation (such as capture of kinetochores by the spindle) may be more affected at a higher temperature when microtubule dynamics is not regulated properly. 
These phenotypes would suggest that Mal3-89R protein might have an elevated activity to stabilize and/or to promote growth of microtubules.

In order to analyze dynamics of microtubules in vivo, we observed microtubules in living cells by a time-lapse microscope. In the wild type cells, growing cytoplasmic microtubules shrunk after a pausing period for 30 seconds on an average.

Microtubules in the mal3-89R mutant were less dynamic. As shown in Fig. 3A and B, the pausing period between growth and shrinkage was 140 seconds. We also noticed that while microtubules in the mal3-89R mutant grew at a rate similar to that in the wild type strain, they shrunk much slower (Fig. 3C). Observation of astral microtubules in mitosis also revealed that the pausing period was much longer in the mal3-89R mutant (Fig. S4). These results indicated that the mal3-89R mutation inhibited disassembly and stabilized microtubules.

\section{Potential binding of Mal3-89R protein to the microtubule lattice}

We next examined localization of Mal3 by visualizing the GFP-tagged protein. Consistently with the previous studies [17, 33], the wild-type Mal3 proteins were found as strong patchy signals at the distal microtubule plus ends and also as very faint signals along the microtubule lattices. In contrast, the signals of the Mal3-89R mutant proteins were found at the ends and much more abundantly along the lattices both in interphase and mitosis (Fig. 4A). We monitored the level of Mal3 and Mal389R mutant proteins. As shown in Fig. 4B, the level of Mal3-89R proteins was comparable to that of the wild type protein, suggesting that abnormal localization of Mal3-89R was not due to an excess of the amount of Mal3-89R proteins.

We observed the Mal3 proteins in living cells. In the wild type cells, the florescent signal from Mal3 protein was found as patches. As Mal3/EB1 concentrates at the tips 
of growing microtubules [17, 28], we speculated that these patches represented the end of growing microtubules. Indeed, the fluorescent signals moved toward the cell tip with comet-like tails (Fig. 4C). Soon after the signals from Mal3-GFP stopped moving, the signals diminished. The Mal3-89R mutant proteins were also found on microtubules. They however remained even after microtubules stopped growth. In mitosis, Mal3-89R also persisted on microtubules composing asters and the spindle (Fig. S5). We speculate that this prolonged interaction of the Mal3-89R with microtubules is a major cause of the longer pausing time before initiation of shrinkage.

\section{Microtubule-dependent phosphorylation of Mal3}

As shown in Fig. 4B, the Mal3-89R mutant proteins were detected as a doublet. We speculated that they might be phosphorylated and tested this possibility. The Mal389R proteins purified by immunoprecipitation with the antibody to Mal3 were treated with the lambda phosphatase. The upper band of the doublet was diminished after the treatment with the phosphatase (Fig. S6), indicating that the Mal3-89R proteins were indeed phosphorylated.

To identify a kinase responsible for phosphorylation of the Mal3-89R protein, we attempted to determine on which cell cycle stage Mal3-89R was phosphorylated. We first tested if Mal3-89R was phosphorylated in mitosis. As shown in Fig. 5A, the upper band of Mal3-89R observed in extracts prepared from cells growing asynchronously, was largely diminished in extracts prepared from cells which were blocked at early mitosis by shifting to the restrictive temperature for the $n d a 3$ mutation. When shifted back to the permissive temperature, the upper band reappeared (Fig 5B). In these experiments we monitored the level of Slp1 that reaches a peak around the onset of anaphase and decreases thereafter [34]. These results 
suggested that Mal3-89R, which was dephosphorylated at early mitosis, was rapidly phosphorylated behind the arrest point of the $n d a 3$ mutation. However, it was also possible that the phosphorylation of Mal3-89R was dependent on the presence of microtubules as most of the microtubules were depolymerized in the nda3 mutant at the restrictive temperature [35]. To test this possibility, we forced microtubuledepolymerization in vivo and examined the status of the Mal3-89R proteins. The $c d c 10$ mutant arrested at $\mathrm{G} 1$ was shifted to $0^{\circ} \mathrm{C}$ to depolymerize microtubules. As shown in Fig. 5C, most of the microtubules were depolymerized in cells after incubation at $0^{\circ} \mathrm{C}$ for 2 hours and reformed soon after incubation at $20^{\circ} \mathrm{C}$. Examination of Mal3-89R proteins by western blot revealed that the upper band of the doublet, which was not detectable in extracts prepared from cells incubated at $0^{\circ} \mathrm{C}$, was reappeared within 20 minutes after incubation at $20^{\circ} \mathrm{C}$ (Fig. 5D). Furthermore, addition of a microtubule poison, CBZ (Carbendazim) prevented both reformation of microtubules and the reappearance of the upper band when the mal3-89R mutant treated at $0^{\circ} \mathrm{C}$ was shifted back to $20^{\circ} \mathrm{C}$ (Fig. $5 \mathrm{E}$ and F). These results illustrated a strong correlation between the phosphorylation of Mal3-89R and the presence of microtubules.

In order to determine the phosphorylation site(s), the Mal3-89R proteins purified by immunoprecipitation were subject to mass spectroscopic analysis. Based on the result (Fig. 6A), the threonine residue at the position 149 (T149) was predicted as a phosphorylation site. Because the region around T149 is very rich in serine/threonine residues (Fig, 6B), it was difficult to precisely determine the phosphorylation site(s). We therefore replaced each of serine/threonine residues between the positions 141 and 155 in the mal3-89R background and tested if the resulting mutant protein was phosphorylated. As shown in Fig. 6B, the intensity of the upper band was reduced in 
some of the mutants. In particular, replacement of S147, T149 and S151 with alanine could effectively suppress the phosphorylation, thought the effect was somehow limited. These results would suggest that the responsible kinase selects the target site sloppily and replacement of the most preferred site (T149) with alanine allows phosphorylation of other sites, or that replacement of S147 (or S151) disrupts a domain structure to define the substrate specificity.

\section{Non-phosphorylatable and phospho-mimetic mal3 mutants}

To address the biological significance of the phosphorylation, we first replaced the three residues S147, T149 and S151 with alanine simultaneously in the mal3-89R background and examined the phenotypes of the resulting mutant (mal3-89R 3A). Like the mal3-89R mutant, the mal3-89R $3 A$ was constructed by replacement of the wild type $\mathrm{mal3}^{+}$gene with the mutated gene and therefore it is identical to wild type except for the presence of the mutation. As shown in Fig. 6C, the simultaneous replacement effectively suppressed the phosphorylation in the mal3-89R background. Observation of the mal3-89R $3 \mathrm{~A}$ mutant indicated that the replacement of the three residues with alanine did not affect the phenotypes associated with the mal3-89R mutation (Fig.7A and C). On the basis of these results, we speculated that the phosphorylation was not the cause of the phenotypes observed in the mal3-89R mutant.

We next constructed a mutant $(\mathrm{mal} 3-3 A)$ in which the three residues of Mal3 were replaced with alanine in the wild type background. By immunoblot with the antibody to Mal3, the wild type Mal3 proteins were detected as a doublet though the intensity of the upper band was weaker than that of Mal3-89R (Fig. 6C). By the same immunoblot, the Mal3-3A proteins were detected as a singlet. We also analyzed the 
wild type Mal3 and Mal3-3A proteins by two-dimensional electrophoresis followed by immunoblot with the antibody to Mal3. As shown in Fig. 6D, two of the three spots of the wild type Mal3 were sensitive to the treatment with the lambda phosphatase, indicating that the wild type protein was phosphorylated at two sites. Because Mal3-3A yielded a single spot, we concluded that the wild type Mal3 was phosphorylated at two of the following three sites; S147, T149 and S151. The previous work indeed reported that Mal3 protein was phosphorylated [17].

Observation of the mal3-3A mutant indicated that microtubule morphology was moderately affected (Fig. 7A). Live cell imaging revealed that while microtubules in the mal3-3A mutant grew at a rate similar to that in the wild type strain, they shrunk slower (Fig. 7B). The results suggested that the mal3-3A mutant made microtubules less dynamic, though the effect on the microtubule morphology was not as strong as that of the mal3-89R mutant. Observation of Mal3-3A tagged with GFP uncovered that, unlike Mal3-89R and Mal3-89R 3A that were found along the lattice, the majority of Mal3-3A was seen as patchy signals (Fig. 7C). The result thereby suggested that the mechanism that localizes Mal3-89R along the lattice was independent from the phosphorylation/dephosphorylation of the cluster of serine/threonine residues of Mal3.

Considering the phenotypes of mal3-3A mutant, we speculated that the phosphorylation normally regulates Mal3 negatively. To test this possibility directly, we replaced the one or two residues of Mal3 (S147 and S151) with glutamate, an amino acid that would mimic phosphorylation and examined them in vitro and in vivo. As shown in Fig. 7D, replacement of S147 to glutamate (Mal3-147E) slightly reduced the activity to promote microtubule assembly in vitro. The simultaneous replacement (Mal3-147/151E) reduced the activity much more significantly. Replacement with 
alanine (Mal3-147/151A, Mal3-147A) did not affect the activity in vitro, indicating that replacement did not disrupt the structure of Mal3 and thus the reduction of the activity of Mal3-147/151E was likely due to a change of the electrostatic property. Furthermore, we measured the binding affinity of Mal3 to microtubules, either by copolymerized or prepolymerized microtubule binding assay. In both assays Mal389R had a greater affinity to microtubules than wild type Mal3, whereas Mal3147/151E had a reduced affinity (Fig. 7E and S7C, Table 2).

In order to examine the effect of the replacement in vivo, the genes encoding Mal3147E and Mal3-147/151E were replaced with the wild type $\mathrm{mal3}^{+}$gene, respectively. The resulting mutants, mal3-147E and mal3-147/151E were examined for their morphology/dynamics of microtubules. While the mutants did not exhibit any phenotypes in interhase, their mitotic spindle elongation was delayed (Fig.7F). The result indicated that replacement of S147/151 with a phospho-mimetic amino acid inhibits the activity of Mal3 in vitro dramatically, but to a much lesser extent in vivo (see discussion). 


\section{Discussion}

Phenotypes of the mal3-89R mutant: Among the four parameters (growth rate, shrinkage rate, catastrophe frequency and rescue frequency) that characterize the dynamic behavior of microtubules, the shrinkage rate is apparently affected by the 89R mutation. We also found that the pausing time is longer at the transition from growth to shrinkage. These phenotypes would suggest that the Mal3-89R proteins confer a higher stability of microtubules. In the recent structural studies fission yeast Mal3 has been localized along the microtubule seam $[15,16,19]$. On the basis of these studies, it has been proposed that Mal3 would reinforce the closure of the tubulin-sheet at the end of the growing microtubules. The mutant protein Mal3-89R may have an elevated activity in this function.

A recent study has explored how Mal3 regulates microtubule dynamics in vitro [36]. The results have illustrated the two activities of Mal3 that are 1) to reduce the shrinkage rate, but not the growth rate and 2) to promote the rescue frequency. These activities are observed in a dose-dependent fashion at a range that exceeds the physiological concentration of Mal3 in fission yeast. It has been proposed that binding of Mal3 to the microtubule lattice plays an important role in exerting these activities. Consistently with this hypothesis, the Mal3-89R proteins that bind the microtubule lattice expressed at a physiological concentration reduce the rate of shrinkage. Although Mal3-89R influences the microtubule assembly in the in vitro system in which Mal3-89R interacts only with purified bovine brain tubulins, we do not exclude the possibility that Ma13-89R influences interaction between other proteins and causes the cellular phenotypes. Mal3-89R mutant protein may prevent its replacement by MT destabilizing factors. This idea is supported by the previous observations that deletion 
of budding yeast EB1 homologue Bim1 and knockdown of Drosophila kinesin-13 decrease catastrophe frequency and prolong the pause state of MTs [37, 38].

The microtubule morphology of the mal3-89R mutant was affected only in mitosis, though the shrinkage rate was reduced and the pausing time was longer in both mitosis and interphase. While we propose that a strong competitor with Mal3-89R functions in interphase, we also speculate that the environment may influence the effect of the mutation on the microtubule morphology. It was shown previously that the rates of polymerization and depolymerization are similar in interphase and mitosis and that the difference in microtubule dynamics is due to an increase in the frequency of transition from growing to shrinking (catastrophe frequency) in mitosis in frog egg extracts $[39,40]$. If microtubule dynamics is regulated in a similar manner in fission yeast, Mal3-89R, that would mask catastrophe by the longer pausing time and lower shrinkage rate, may impact more severely mitotic microtubules.

Phosphorylation of Mal3: Mal3-89R is highly phosphorylated at the serine/threonine-rich region connecting $\mathrm{CH}$ and $\mathrm{EB} 1$-like $\mathrm{C}$-terminal motif domains. Upon depolymerization of microtubules, the phosphorylation was disappeared in the mal3-89R mutant. The observation suggests that Mal3-89R is phosphorylated while it is associates with microtubules and dephosphorylated after dissociation from microtubules. Simultaneous replacement of three residues (S147, T149 and S151) with alanine ( $3 A$ mutation) in the serine/threonine-rich region resulted in disappearance of the upper band, but it did not affect the phenotypes associated with the mal3-89R mutation, indicating that the phosphorylation is not a cause of the phenotypes. The $3 A$ mutation resulted in disappearance of the upper band not only in the mal3-89R mutant, but also in the wild type strain, indicating that the wild type 
Mal3 is also phosphorylated. We confirmed the phosphorylation of the wild type Mal3 by 2D gel electrophoresis followed by the treatment with the lambda phosphatase. The phosphorylation is readily detectable in the mal3-89R mutant. Unlike the wild type Mal3 that only accumulates at the growing ends, the Mal3-89R proteins associate with the entire length of microtubules. It is likely that a larger part of the Mal3-89R would remain bound to microtubules and thus be much more phosphorylated.

Analysis of phospho-mimetic mal3 mutant protein (Mal3-147/151E) in vitro indicated that the mutant had a reduced affinity to microtubules and could not promote the microtubule assembly. These results indicate that the phosphorylation normally regulates Mal3 negatively. The phenotypes of the non-phosphorylatable mutant (mal3-3A) and phospho-mimetic mal3 mutant in vivo also support this notion. The mal3-3A mutation reduced the rate of shrinkage (thus stabilized microtubules). We also found that the time for separation of the poles was longer (thus likely the elongation of the mitotic spindle was slower) in the mal3-147/151E mutant. Considering the phenotype of cells lacking Mal3 (short interphase microtubules; Fig. $2 \mathrm{~A} ;[17,20,41])$, the phenotype of the mal3-147/151E mutant was very modest. Regulation of Mal3 is complex and a factor may assist Mal3-147/151E in vivo. It has been demonstrated that the serine/threonine-rich region of Mal3 is involved in binding to microtubules $[10,15]$. A recent study has further elucidated a mechanism that regulates interaction between EB1 and microtubules. Removal of the negatively charged C-terminal domain from EB1 results in stable association with the microtubule lattice in vitro, suggesting that electrostatic repulsion prevents stable binding of EB1 with the lattice [42]. The phosphorylation at the serine/threonine-rich region would simply add negative charge or change the conformation to further 
reduce the microtubule-binding activity. We propose the mal3-89R mutation can overcome the negative regulation by the phosphorylation.

The biological significance of phosphorylation at a cluster of serine residues in the linker connecting the $\mathrm{CH}$ and $\mathrm{EB} 1-$ like $\mathrm{C}$-terminal motif domains has first been demonstrated in budding yeast. Phosphorylation of Bim1 (a homolog of Mal3/EB1) by Aurora kinase during anaphase promotes dissociation from microtubules [43]. We show here that the analogous linker domain of the fission yeast Mal3 is phosphorylated. While Mal3 might be phosphorylated by Ark1 (the fission yeast Aurora kinase), we would not exclude a possibility that another kinase is also responsible for phosphorylation of Mal3. The phosphorylation was recovered within 60 minutes at $20^{\circ} \mathrm{C}$ after release of the cdc10-G1 block. Most of the cells under this experimental condition would be in interphase. Regulation of microtubules in fission yeast may be different from that of budding yeast in which major spatial cues are dependent on septins and actin, but not microtubules [44]. 


\section{Materials and Methods}

\section{Yeast strains, Media, and Transformation}

The Schizosaccharomyces pombe strains are listed in Supplementary table 1. The strains were grown in YES (yeast extract with supplement) media and EMM synthetic minimal media with appropriate nutrient supplements as described by Moreno et al. [45]. All yeast transformations were carried out by lithium acetate methods [46, 47]. For synchronization, $n d a 3-K M 311$ cold-sensitive mutant were grown in YPD media at $32^{\circ} \mathrm{C}$, a permissive condition, and then incubated at $20^{\circ} \mathrm{C}$ for 8 hours, a restrictive condition. $c d c 10-M 17$ and $c d c 25-22$ temperature-sensitive mutants were grown at $26^{\circ} \mathrm{C}$, a permissive condition, and then incubated at $36^{\circ} \mathrm{C}$ for 4 hours, a restrictive condition.

\section{Mutagenesis of $\mathrm{mal3}^{+}$gene}

The $\mathrm{mal3}^{+}$gene was amplified by PCR using the forward primer 5'-mal3 [5'GGGGGGGCATATG(NdeI)TCTGAATCTCGGCAAGAGC-3'] and the reverse primer 3'-mal3 [5'-GGGGATCC(BamHI)TTAAAACGTGATATTCTCATCG-3']. In order to introduce mutations, PCR was performed in the presence of $1.25 \mathrm{mM} \mathrm{Mg}^{2+}$ and $0.25 \mathrm{mM} \mathrm{Mn}^{2+}$. The resulting fragments were digested with NdeI and BamHI and then cloned into pREP81 to construct a pool of the mal3 mutants.

\section{Construction of mal3-GFP, mal3-89R and other mal3 mutants}

The homologous recombination-based method was used to tag endogenous mal3 with GFP at its carboxyl terminus and to generate the mal3 mutants. 
The mal3-89R mutation was introduced into a cloned genomic DNA fragment (3025 bp franked by $X h o I$ and $K p n I$ restriction sites). The resulting fragments were used to transform a haploid strain in which the $\mathrm{mal}^{+}$gene was replaced with the $\mathrm{rra}^{+}$gene. The ura ${ }^{-}$transformants were selected and the presence of the mal3-89R mutation was confirmed by genomic DNA sequencing. Other mutants in which the serine/threonine residues in the serine/threonine-rich region of Mal3 were replaced with alanine, were constructed similarly.

\section{Fluorescent Microscopy}

For time-lapse observation, living cells expressing Mal3-GFP from the native locus or GFP-Atb2 from the native $n d a 3$ promoters integrated at the lys 1 locus [48] were grown to mid- $\log$ phase in $\mathrm{EMM}$ medium at $30^{\circ} \mathrm{C}$ and transferred to $35-\mathrm{mm}$ glassbottom dishes (MatTek) coated with $0.2 \mathrm{mg} / \mathrm{ml}$ lectin (Sigma). Fluorescence microscope images were obtained by the Delta Vision microscope system (Applied Precision, Inc.) and set up in a temperature-controlled room as previously described [49]. This microscope system is based on an inverted fluorescence microscope (IX70; Olympus) equipped with a charge-coupled device (CoolSNAP HQ; Photometrics). The objective lens used was an oil immersion Plan-Apochromat 60× NA 1.4 lens (Olympus). A stack of nine slices $(0.3 \mu \mathrm{m}$ distance between planes) was projected with softWoRx software (Applied Precision, Inc.) using a maximum intensity method in Fig. 3, 4C, 7D, S3C and D, S4 and S5. The velocity of microtubule plus-end growth and shrinkage was analyzed with softWoRx software (Applied Precision, Inc.) by measuring the distance of microtubule plus ends between two time points in Fig. $3 \mathrm{C}$ and $7 \mathrm{~B}$. 


\section{Microtubule-polymerization assay}

Tubulin was isolated from bovine brain as described [50]. DNA fragment of mal3 and mutants were cloned into pET30a. Mal3 with an N-terminal His-tag was expressed in BL21 (DE3) and purified with Ni-NTA Superflow Cartride (Qiagen) and HiTrapQ (GE Healthcare). Tubulin polymerization assay was performed as previously described [14]. Briefly, $5 \mu \mathrm{M}$ of tubulin was added to the reaction mixture containing $2 \mu \mathrm{M}$ Mal3 in PMEM buffer (50 mM K-PIPES, 50 mM MES, 1 mM EGTA, 1 mM $\left.\mathrm{MgCl}_{2}, 1 \mathrm{mM} \mathrm{GTP},[\mathrm{pH} 6.8]\right)$. The turbidity was recorded as the optical absorbance at $350 \mathrm{~nm}$ using a JASCO V-630 Bio spectrophotometer in 15 second intervals at $37^{\circ} \mathrm{C}$. The wild type and various mutant Mal3 proteins were tagged with $6 \mathrm{xHis}$, expressed and purified from bacteria. The final preparation of each protein used in the assay was shown in Fig. S7A.

\section{Microtubule binding assays}

For the copolymerized microtubule binding assay, $60 \mu 1$ mixtures of $5 \mu \mathrm{M}$ of tubulin and $0.5-12 \mu \mathrm{M}$ Mal3 were copolymerized in PMEM buffer containing $100 \mathrm{mM} \mathrm{NaCl}$ at $37^{\circ} \mathrm{C}$ for 30 minutes. For the prepolymerized microtubule binding assay, purified tubulin was polymerized in PMEM buffer with $10 \mu \mathrm{M}$ Taxol at a concentration of $50 \mu \mathrm{M}$ at $30^{\circ} \mathrm{C}$ for 30 minutes as described [12]. Mixtures $(60 \mu 1)$ of taxol-stabilized microtubules and 0.5-12 $\mu \mathrm{M}$ Mal3 were incubated in PMEM buffer containing 100 $\mathrm{mM} \mathrm{NaCl}$ at $37^{\circ} \mathrm{C}$ for 30 minutes.

All samples $(50 \mu 1)$ were centrifuged through a $40 \%$ glycerol cushion $(250 \mu 1)$ for $7 \mathrm{~min}$ at $35^{\circ} \mathrm{C}$ at $70,000 \mathrm{rpm}$ in a TLA100.3 rotor (Beckman). After centrifugation, pellet and supernatant were resuspended in SDS-PAGE loading buffer and analyzed by SDS-PAGE. Polyacrylamide gels were stained with Coomassie Brilliant Blue and 
the images of the gels were recorded using LAS-4000 image reader (Fuji Photo Film). Protein bands were quantified using Multi Gauge 3.2 software (Fuji Photo Film). The nonlinear fit of the all data were calculated using the program PRISM (GraphPad software) as described [15].

\section{Biochemistry}

Protein extraction and western blotting were performed as described [34]. The antiMal3 rabbit polyclonal antibody was raised against His-Mal3 in this study. The quantitative analysis was performed with ImageJ software (NIH) in Fig. 4B. Lambda phosphatase (New England Biolabs) treatment of immunoprecipitates was performed according to the manufacture's instructions. For tow-dimensional electrophoresis, Mal3 immunoprecipitates were separated by isoelectric focusing on $\mathrm{pH}$ 3-6 ReadyStrip IPG strips by using the PROTEAN IEF cell (Bio-Rad laboratories) according to the manufacture's instructions.

\section{MS spectrometry}

A mal3 deletion mutant was transformed with pREP1-mal3 or pREP1-mal3-89R. Protein extraction was performed as described [34]. Supernatants were incubated with anti-Mal3 antibody (generated against His-Mal3 in this study) for 2 hours at $4{ }^{\circ} \mathrm{C}$ before addition of priteinG-Agarose (Pierce) for a further 1 hour. Beads are washed five times with lysis buffer, and boiled in SDS sample buffuer. Immunoprecipitated samples were separated on SDS-PAGE gel and the region of the gels containing Mal3 proteins from about $40 \mathrm{~K}$ to $30 \mathrm{~K}$ were cut and analyzed as described [51]. The obtained spectra were searched against GeneDB (pombe) database by Mascot (Matrux 
Science). Some peaks were further assigned manually as described in the legend for Fig. 6A. 


\section{Conflicts of interest}

The authors declare that there are no conflicts of interest.

\section{Acknowledgements}

The authors greatly appreciate Keith Gull (University of Oxford, United Kingdom)

for providing the TAT-1 antibody, and members of the Matsumoto lab for discussion.

The plasmid for tagging endogenous mal3 with GFP was supplied by National

BioResource Project (http://yeast.lab.nig.ac.jp/). This work was supported by grants

from Ministry of Education, Culture, Sports, Science and Technology of Japan (to T.

M. and Y. H.) and a grant from Japan Society for the Promotion of Science (to Y. C.). 


\section{References}

[1] A. Desai, T.J. Mitchison, Microtubule polymerization dynamics, Annu Rev Cell Dev Biol 13 (1997) 83-117.

[2] T. Mitchison, M. Kirschner, Dynamic instability of microtubule growth, Nature 312 (1984) 237-242.

[3] D.R. Drummond, R.A. Cross, Dynamics of interphase microtubules in Schizosaccharomyces pombe, Curr Biol 10 (2000) 766-775.

[4] Y. Mimori-Kiyosue, S. Tsukita, "Search-and-capture" of microtubules through plus-end-binding proteins (+TIPs), J Biochem 134 (2003) 321-326.

[5] S.C. Schuyler, D. Pellman, Microtubule "plus-end-tracking proteins": The end is just the beginning, Cell 105 (2001) 421-424.

[6] E.E. Morrison, Action and interactions at microtubule ends, Cell Mol Life Sci 64 (2007) 307-317.

[7] A. Akhmanova, M.O. Steinmetz, Tracking the ends: a dynamic protein network controls the fate of microtubule tips, Nat Rev Mol Cell Biol 9 (2008) 309-322.

[8] L.K. Su, M. Burrell, D.E. Hill, J. Gyuris, R. Brent, R. Wiltshire, J. Trent, B. Vogelstein, K.W. Kinzler, APC binds to the novel protein EB1, Cancer Res 55 (1995) 2972-2977.

[9] G. Lansbergen, A. Akhmanova, Microtubule plus end: a hub of cellular activities, Traffic 7 (2006) 499-507.

[10] Y. Komarova, C.O. De Groot, I. Grigoriev, S.M. Gouveia, E.L. Munteanu, J.M. Schober, S. Honnappa, R.M. Buey, C.C. Hoogenraad, M. Dogterom, G.G. 
Borisy, M.O. Steinmetz, A. Akhmanova, Mammalian end binding proteins control persistent microtubule growth, J Cell Biol 184 (2009) 691-706.

[11] K.C. Slep, R.D. Vale, Structural basis of microtubule plus end tracking by XMAP215, CLIP-170, and EB1, Mol Cell 27 (2007) 976-991.

[12] I. Hayashi, M. Ikura, Crystal structure of the amino-terminal microtubulebinding domain of end-binding protein 1 (EB1), J Biol Chem 278 (2003) $36430-36434$.

[13] R.R. Wei, J. Al-Bassam, S.C. Harrison, The Ndc80/HEC1 complex is a contact point for kinetochore-microtubule attachment, Nat Struct Mol Biol 14 (2007) 54-59.

[14] I. Hayashi, A. Wilde, T.K. Mal, M. Ikura, Structural basis for the activation of microtubule assembly by the EB1 and p150Glued complex, Mol Cell 19 (2005) 449-460.

[15] A. des Georges, M. Katsuki, D.R. Drummond, M. Osei, R.A. Cross, L.A. Amos, Mal3, the Schizosaccharomyces pombe homolog of EB1, changes the microtubule lattice, Nat Struct Mol Biol 15 (2008) 1102-1108.

[16] B. Vitre, F.M. Coquelle, C. Heichette, C. Garnier, D. Chretien, I. Arnal, EB1 regulates microtubule dynamics and tubulin sheet closure in vitro, Nat Cell Biol 10 (2008) 415-421.

[17] K.E. Busch, D. Brunner, The microtubule plus end-tracking proteins mal3p and tiplp cooperate for cell-end targeting of interphase microtubules, Curr Biol 14 (2004) 548-559.

[18] P. Bieling, L. Laan, H. Schek, E.L. Munteanu, L. Sandblad, M. Dogterom, D. Brunner, T. Surrey, Reconstitution of a microtubule plus-end tracking system in vitro, Nature 450 (2007) 1100-1105. 
[19] L. Sandblad, K.E. Busch, P. Tittmann, H. Gross, D. Brunner, A. Hoenger, The Schizosaccharomyces pombe EB1 homolog Mal3p binds and stabilizes the microtubule lattice seam, Cell 127 (2006) 1415-1424.

[20] J.D. Beinhauer, I.M. Hagan, J.H. Hegemann, U. Fleig, Mal3, the fission yeast homologue of the human APC-interacting protein EB-1 is required for microtubule integrity and the maintenance of cell form, J Cell Biol 139 (1997) $717-728$.

[21] H. Browning, D.D. Hackney, P. Nurse, Targeted movement of cell end factors in fission yeast, Nat Cell Biol 5 (2003) 812-818.

[22] D. Brunner, P. Nurse, CLIP170-like tip1p spatially organizes microtubular dynamics in fission yeast, Cell 102 (2000) 695-704.

[23] K.E. Busch, J. Hayles, P. Nurse, D. Brunner, Tea2p kinesin is involved in spatial microtubule organization by transporting tip1p on microtubules, Dev Cell 6 (2004) 831-843.

[24] A. Kerres, C. Vietmeier-Decker, J. Ortiz, I. Karig, C. Beuter, J. Hegemann, J. Lechner, U. Fleig, The fission yeast kinetochore component Spc7 associates with the EB1 family member Mal3 and is required for kinetochore-spindle association, Mol Biol Cell 15 (2004) 5255-5267.

[25] A. Kerres, V. Jakopec, U. Fleig, The conserved Spc7 protein is required for spindle integrity and links kinetochore complexes in fission yeast, Mol Biol Cell 18 (2007) 2441-2454.

[26] K. Asakawa, M. Toya, M. Sato, M. Kanai, K. Kume, T. Goshima, M.A. Garcia, D. Hirata, T. Toda, Mal3, the fission yeast EB1 homologue, cooperates with Bub1 spindle checkpoint to prevent monopolar attachment, EMBO Rep 6 (2005) 1194-1200. 
[27] M.W. Robinson, N. McFerran, A. Trudgett, L. Hoey, I. Fairweather, A possible model of benzimidazole binding to beta-tubulin disclosed by invoking an inter-domain movement, J Mol Graph Model 23 (2004) 275-284.

[28] M.A. Garcia, L. Vardy, N. Koonrugsa, T. Toda, Fission yeast chTOG/XMAP215 homologue Alp14 connects mitotic spindles with the kinetochore and is a component of the Mad2-dependent spindle checkpoint, Embo J 20 (2001) 3389-3401.

[29] A. Unsworth, H. Masuda, S. Dhut, T. Toda, Fission yeast kinesin-8 Klp5 and Klp6 are interdependent for mitotic nuclear retention and required for proper microtubule dynamics, Mol Biol Cell 19 (2008) 5104-5115.

[30] L. Chang, K.L. Gould, Sid4p is required to localize components of the septation initiation pathway to the spindle pole body in fission yeast, Proc Natl Acad Sci U S A 97 (2000) 5249-5254.

[31] J.L. Morrell, G.C. Tomlin, S. Rajagopalan, S. Venkatram, A.S. Feoktistova, J.J. Tasto, S. Mehta, J.L. Jennings, A. Link, M.K. Balasubramanian, K.L. Gould, Sid4p-Cdc11p assembles the septation initiation network and its regulators at the S. pombe SPB, Curr Biol 14 (2004) 579-584.

[32] K. Aoki, Y. Nakaseko, K. Kinoshita, G. Goshima, M. Yanagida, CDC2 phosphorylation of the fission yeast dis1 ensures accurate chromosome segregation, Curr Biol 16 (2006) 1627-1635.

[33] K. Asakawa, K. Kume, M. Kanai, T. Goshima, K. Miyahara, S. Dhut, W.W. Tee, D. Hirata, T. Toda, The V260I mutation in fission yeast alpha-tubulin Atb2 affects microtubule dynamics and EB1-Mal3 localization and activates the Bub1 branch of the spindle checkpoint, Mol Biol Cell 17 (2006) 14211435. 
[34] A.E. Ikui, K. Furuya, M. Yanagida, T. Matsumoto, Control of localization of a spindle checkpoint protein, Mad2, in fission yeast, J Cell Sci 115 (2002) 16031610.

[35] Y. Hiraoka, T. Toda, M. Yanagida, The NDA3 gene of fission yeast encodes beta-tubulin: a cold-sensitive nda3 mutation reversibly blocks spindle formation and chromosome movement in mitosis, Cell 39 (1984) 349-358.

[36] M. Katsuki, D.R. Drummond, M. Osei, R.A. Cross, Mal3 masks catastrophe events in Schizosaccharomyces pombe microtubules by inhibiting shrinkage and promoting rescue, J Biol Chem 284 (2009) 29246-29250.

[37] J.S. Tirnauer, E. O'Toole, L. Berrueta, B.E. Bierer, D. Pellman, Yeast Bim1p promotes the G1-specific dynamics of microtubules, J Cell Biol 145 (1999) 993-1007.

[38] V. Mennella, G.C. Rogers, S.L. Rogers, D.W. Buster, R.D. Vale, D.J. Sharp, Functionally distinct kinesin-13 family members cooperate to regulate microtubule dynamics during interphase, Nat Cell Biol 7 (2005) 235-245.

[39] L.D. Belmont, A.A. Hyman, K.E. Sawin, T.J. Mitchison, Real-time visualization of cell cycle-dependent changes in microtubule dynamics in cytoplasmic extracts, Cell 62 (1990) 579-589.

[40] F. Verde, M. Dogterom, E. Stelzer, E. Karsenti, S. Leibler, Control of microtubule dynamics and length by cyclin A- and cyclin B-dependent kinases in Xenopus egg extracts, J Cell Biol 118 (1992) 1097-1108.

[41] C.R. Chen, J. Chen, E.C. Chang, A conserved interaction between Moe1 and Mal3 is important for proper spindle formation in Schizosaccharomyces pombe, Mol Biol Cell 11 (2000) 4067-4077. 
[42] R.M. Buey, R. Mohan, K. Leslie, T. Walzthoeni, J.H. Missimer, A. Menzel, S. Bjelic, K. Bargsten, I. Grigoriev, I. Smal, E. Meijering, R. Aebersold, A. Akhmanova, M.O. Steinmetz, Insights into EB1 structure and the role of its Cterminal domain for discriminating microtubule tips from the lattice, Molecular biology of the cell 22 (2011) 2912-2923.

[43] T. Zimniak, K. Stengl, K. Mechtler, S. Westermann, Phosphoregulation of the budding yeast EB1 homologue Bim1p by Aurora/Ipl1p, J Cell Biol 186 (2009) 379-391.

[44] F. Chang, S.G. Martin, Shaping fission yeast with microtubules, Cold Spring Harb Perspect Biol 1 (2009) a001347.

[45] S. Moreno, A. Klar, P. Nurse, Molecular genetic analysis of fission yeast Schizosaccharomyces pombe, Methods Enzymol 194 (1991) 795-823.

[46] K. Okazaki, N. Okazaki, K. Kume, S. Jinno, K. Tanaka, H. Okayama, Highfrequency transformation method and library transducing vectors for cloning mammalian cDNAs by trans-complementation of Schizosaccharomyces pombe, Nucleic Acids Res 18 (1990) 6485-6489.

[47] D. Gietz, A. St Jean, R.A. Woods, R.H. Schiestl, Improved method for high efficiency transformation of intact yeast cells, Nucleic Acids Res 20 (1992) 1425.

[48] H. Masuda, T. Toda, R. Miyamoto, T. Haraguchi, Y. Hiraoka, Modulation of Alp4 function in Schizosaccharomyces pombe induces novel phenotypes that imply distinct functions for nuclear and cytoplasmic gamma-tubulin complexes, Genes Cells 11 (2006) 319-336. 
[49] T. Haraguchi, D.Q. Ding, A. Yamamoto, T. Kaneda, T. Koujin, Y. Hiraoka, Multiple-color fluorescence imaging of chromosomes and microtubules in living cells, Cell Struct Funct 24 (1999) 291-298.

[50] A. Hyman, D. Drechsel, D. Kellogg, S. Salser, K. Sawin, P. Steffen, L. Wordeman, T. Mitchison, Preparation of modified tubulins, Methods Enzymol 196 (1991) 478-485.

[51] R.S. Nozawa, K. Nagao, H.T. Masuda, O. Iwasaki, T. Hirota, N. Nozaki, H. Kimura, C. Obuse, Human POGZ modulates dissociation of HP1alpha from mitotic chromosome arms through Aurora B activation, Nat Cell Biol 12 (2010) 719-727.

[52] A. Sali, T.L. Blundell, Comparative protein modelling by satisfaction of spatial restraints, J Mol Biol 234 (1993) 779-815.

[53] Y. Nakase, K. Fukuda, Y. Chikashige, C. Tsutsumi, D. Morita, S. Kawamoto, M. Ohnuki, Y. Hiraoka, T. Matsumoto, A defect in protein farnesylation suppresses a loss of Schizosaccharomyces pombe tsc2+, a homolog of the human gene predisposing to tuberous sclerosis complex, Genetics 173 (2006) $569-578$. 


\section{Legends for Figures and Tables}

Fig. 1. Phenotype of the mal3-89R mutant. (A) Mutation site of mal3-89R. The mutation site is shown with a domain structure of Mal3 and the amino acid sequences of other members of the EB1 family. Amino acids conserved among all the members are shaded in black and in more than three in gray. (B) Location of the mal3-89R mutation. Q89R and conserved hydrophobic residues are labeled and shown as balland-stick. The model was built using MODELLER [52] and represented by PyMOL Molecular Graphic System (http://www.pymol.org). (C) Effect on in vitro microtubule assembly assay. Wild type Mal3 and Mal3-89R proteins were examined for their ability to promote the microtubule-assembly.

Fig. 2. Microtubules in the mal3-89R mutant. (A) The $\alpha$-tubulin was tagged with GFP (GFP-Atb2) in each strain and morphology of microtubules was observed in interphase (I) and mitosis (M) under a fluorescent microscope. (B and C) Frequency of abnormal microtubules. The samples observed in (A) were categorized by counting more than 250 mitotic cells (B). (C) Morphology of microtubules in mitosis. Mitotic microtubules were stained with the antibody to $\alpha$-tubulin, TAT-1. The phenotypes of abnormal microtubules were observed in the mal3-89R strain, which was expressing non-tagged $\alpha$-tubulin. The bar indicates $5 \mu \mathrm{m}$.

Fig. 3. Microtubule dynamics in the mal3-89R mutant. (A) The $\alpha$-tubulin was tagged with GFP (GFP-Atb2) in each strain and dynamics of microtubules was observed by live cell imaging. Each panel shows a 10-sec-interval series of maximal projections of the indicated strains. The black arrowheads highlight microtubule tips that will pause 
for the period indicated by the boxes around the frames. Thus, the microtubule in the example of a wild-type cell pauses at the right tip for $30 \mathrm{sec}$, while in the mal3-89R mutant, the microtubule pauses for more than $100 \mathrm{sec}$. Numbers in the corner of panels indicate relative timing in seconds. The white arrowheads indicate microtubule tips in the phase of shrinkage. (B and C) The live cell images were analyzed for statistics for the pausing time (B) and growth and shrinkage rates (C). The lines are the median and the bars indicate the inter-quartile range (nonparametric MannWhitney $U$ test). 18 cells and 30 cells were measured for statistics for the pausing time and for growth and shrinkage rates. The bar indicates $5 \mu \mathrm{m}$.

Fig. 4. Characterization of Mal3-89R protein. (A) The wild type Mal3 and the mutant were tagged with GFP and examined under a fluorescent microscope. The images were taken and processed in the same way. (B) The cell extracts prepared from the indicated strains were subjected to immunoblot analysis with an antibody to Mal3, Rheb [53] and TAT-1 (antibody to $\alpha$-tubulin) as a loading control. (C) The strains shown in (A) were analyzed by live cell imaging. Each panel shows a 10-sec-interval series of maximal projections of the indicated strains. The white arrowheads highlight the predicted growing ends of microtubule that will pause for the period indicated by the white boxes around the frames. The bar indicates $5 \mu \mathrm{m}$.

Fig. 5. Phosphorylation of Mal3-89R. (A and B) The nda3-KM311 mutants were grown at $32^{\circ} \mathrm{C}$, and then incubated at $20^{\circ} \mathrm{C}$ for blocking at early mitosis. For release experiment, the cells were re-incubated at $32^{\circ} \mathrm{C}$ and collected every 3 minutes (B). (C and D) The $c d c 10-M 17$ mutants were grown at $26^{\circ} \mathrm{C}$, and then incubated at $36^{\circ} \mathrm{C}$ for blocking at G1 phase. Furthermore, the cells were incubated on ice. For release 
experiment, the cells were re-incubated at $20^{\circ} \mathrm{C}$ and collected at the indicated time. (E and F) The mal3-89R mutant cells were grown at $30^{\circ} \mathrm{C}$, and then incubated on ice. For re-assembling of microtubules, the cells were re-incubated at $20^{\circ} \mathrm{C}$ with or without CBZ, microtubule-destabilizing drug, and collected at the indicated time. (C and E) Immuno-staining of microtubules with the antibody to $\alpha$-tubulin, TAT-1, was performed as previously described [34].

Fig. 6. Identification of Mal3-89R phosphorylation sites. (A) Identification of Mal389R phosphorylation sites by mass spectrometric analysis. The MS/MS spectra of the tryptic phosphopeptide amino acids $142-152$ of Mal3 obtained by collision-induced dissociation of the $[\mathrm{M}+2 \mathrm{H}]^{2+}$ precursor ions, $m / z 696.9$ is shown. Intense $[\mathrm{M}+2 \mathrm{H}-$ $\left.2 \mathrm{H}_{2} \mathrm{O}\right]^{2+}$ ion $(m / z 679.2)$ and $\left[\mathrm{M}+2 \mathrm{H}-2 \mathrm{H}_{2} \mathrm{O}-98\right]^{2+}$ ion $(m / z 630.5)$, due to $\mathrm{H}_{2} \mathrm{O}$ and/or neutral loss $\left(\mathrm{H}_{2} \mathrm{O}+\right.$ phosphate $)$ of precursor ions were observed, indicating that the potential phosphorylated residue is serine or threonine. The evident ion fragment at $\mathrm{m} / \mathrm{z} 760.2$ is assignable to $\mathrm{y} 6$ (boxed) and the following y series assignments are reasonable when the peptide is phosphorylated at Thr149. (B) Cells expressing the indicated mutant alleles of $\mathrm{mal} 3$ were grown at $32^{\circ} \mathrm{C}$. (C) The cell extracts prepared from the indicated strains were subjected to immunoblot analysis with antibodies to Mal3, Rheb and TAT-1. (D) The samples prepared as in (C) were analyzed by twodimensional electrophoresis followed by immunoblot with the antibody to Mal3.

Fig. 7. Phenotypes of non-phosphorylatable and phospho-mimetic mal3 mutants. (A) The $\alpha$-tubulin was tagged with GFP (GFP-Atb2) in each strain and morphology of microtubules was observed in mitosis under a fluorescent microscope. The samples were categorized as shown in Fig2B. (B) The live cell images were analyzed for 
growth and shrinkage rates. The lines are the median and the bars indicate the interquartile range (nonparametric Mann-Whitney U test). 30 cells were measured for statistics for growth and shrinkage rates. (C) The Mal3-3A and Mal3-89R3A mutants were tagged with GFP and examined under a fluorescent microscope. The images were taken and processed in the same way. The bar indicates $5 \mu \mathrm{m}$. (D) Effect on in vitro microtubule assembly assay. Wild type Mal3, phospho-mimetic and nonphosphorylatable mutant proteins were examined for their ability to promote the microtubule-assembly. (E) Effect on in vitro microtubule binding assay. Wild type Ma13, Ma13-89R and phospho-mimetic mutant proteins were examined for the binding affinity to copolumerized (left) or prepolumerized (taxol-stabilized; right) microtubules. (F) Wild-type, mal3-89R and phospho-mimetic mutant strains, each of which was expressing GFP-tagged Cnp1 (as a marker for centromere) and mCherrytagged Sad1, were observed by a time-lapse microscope. The images were taken every 2 minutes and the time required for mitosis (defined as time between SPB separation and complete centromere separation) was determined. The lines are the median and the bars indicate the inter-quartile range ( $n=11$, nonparametric MannWhitney $U$ test).

Table 1. Stability of mini-chromosome. Stability of an artificial mini-chromosome (Ch16) was examined in a wild-type strain, a strain deleted for $\mathrm{mal3}^{+}$gene and a mal3-89R mutant at 26 or $36^{\circ} \mathrm{C}$ as described previously [32].

Table 2. Binding affinity of Mal3 mutant proteins. The maximum specific binding $\left(B_{\max }\right)$ and equilibrium dissociation constant $\left(\mathrm{K}_{\mathrm{d}}\right)$ were calculated based on the data shown in Fig. 7E by a fitting program PRISM (GraphPad software). 
A

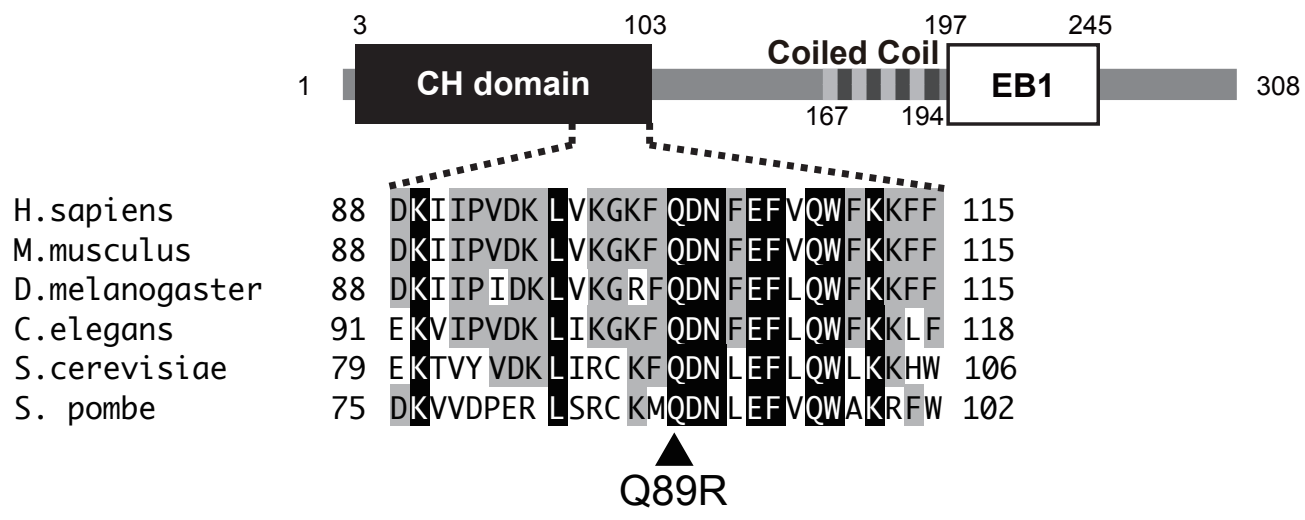

B

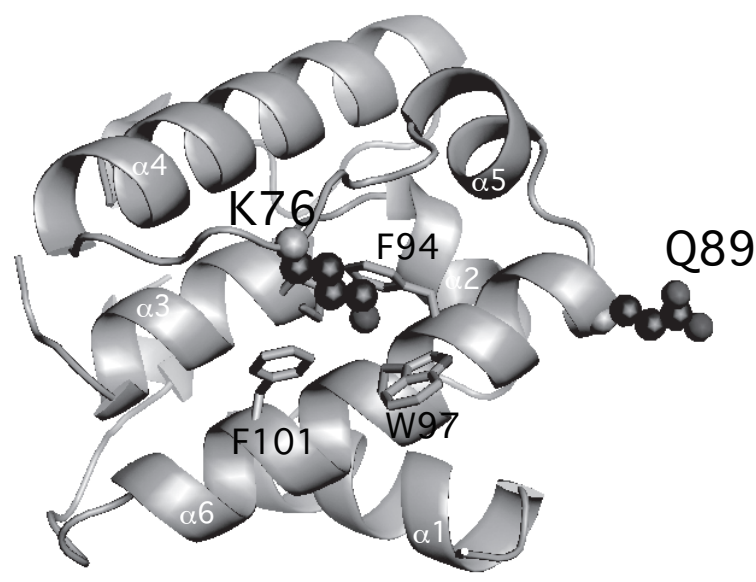

C

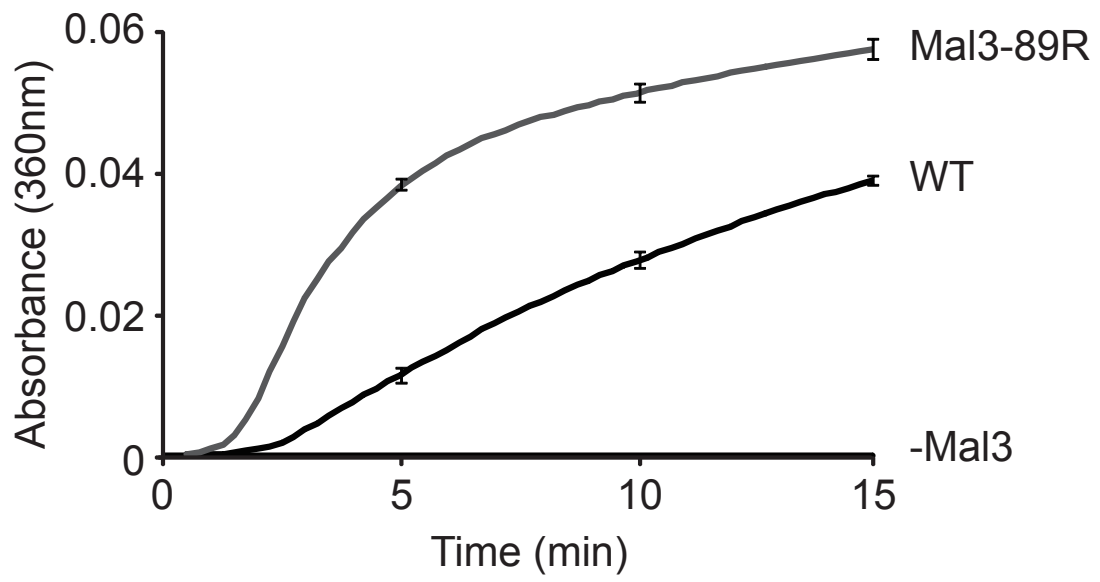


A

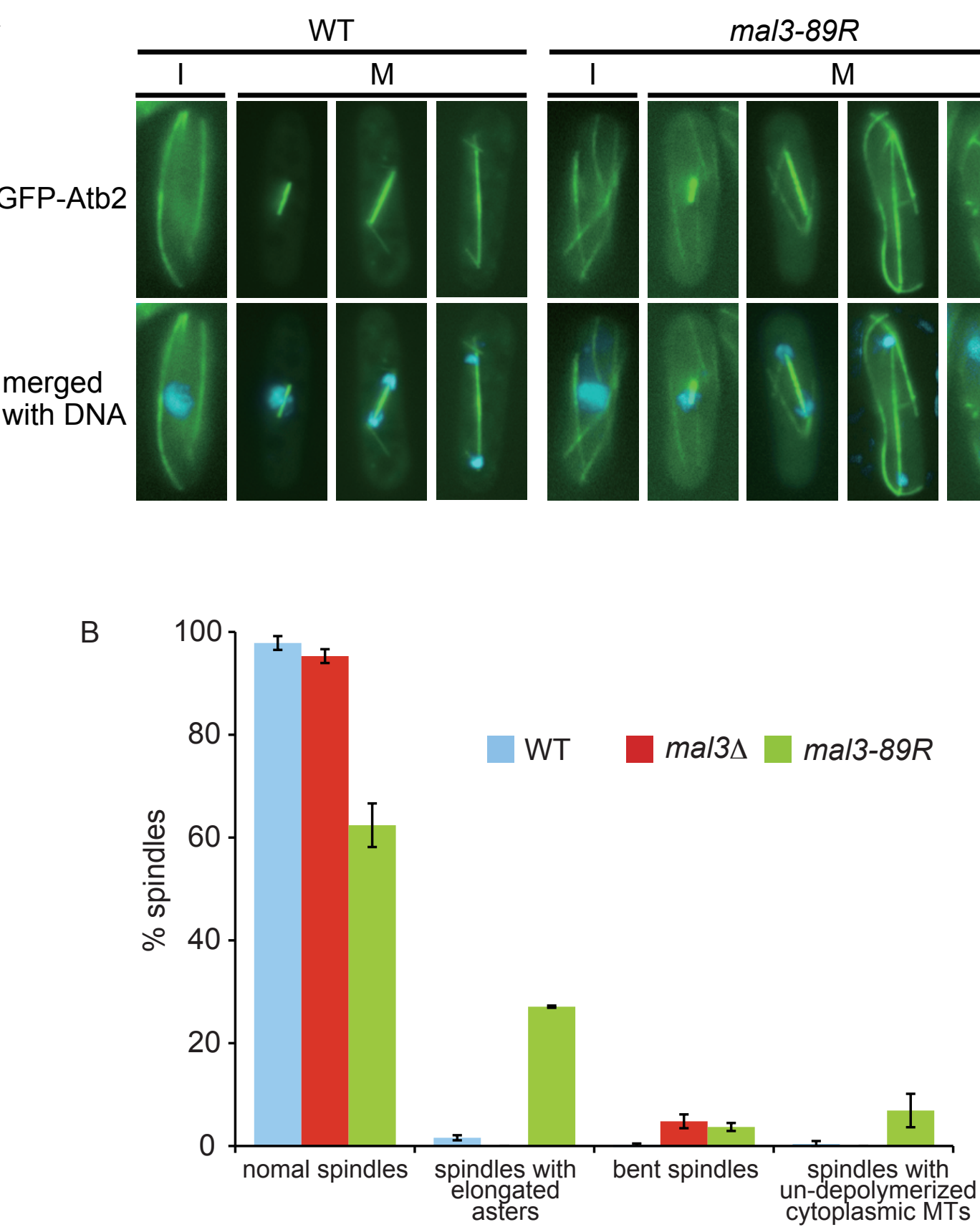

C

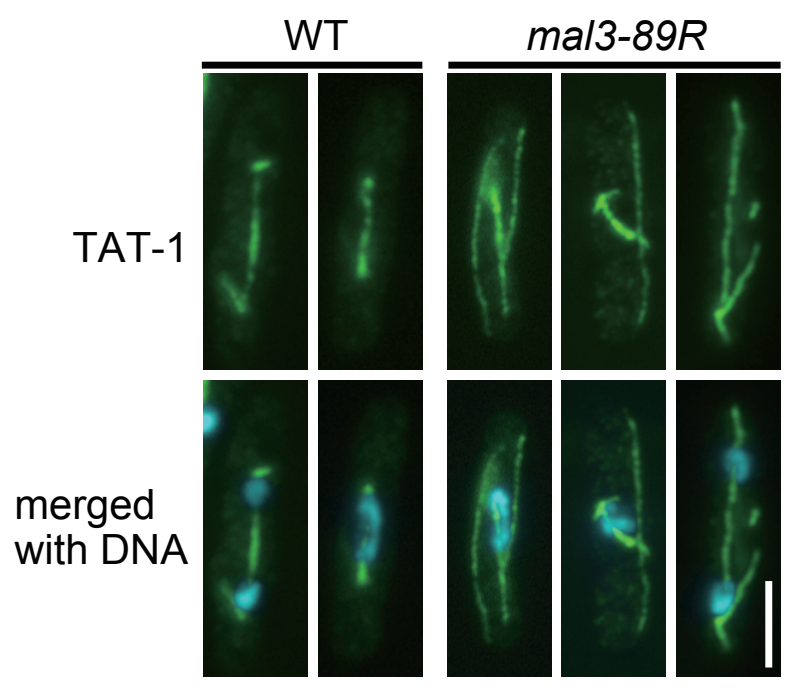

Fig. 2 
A

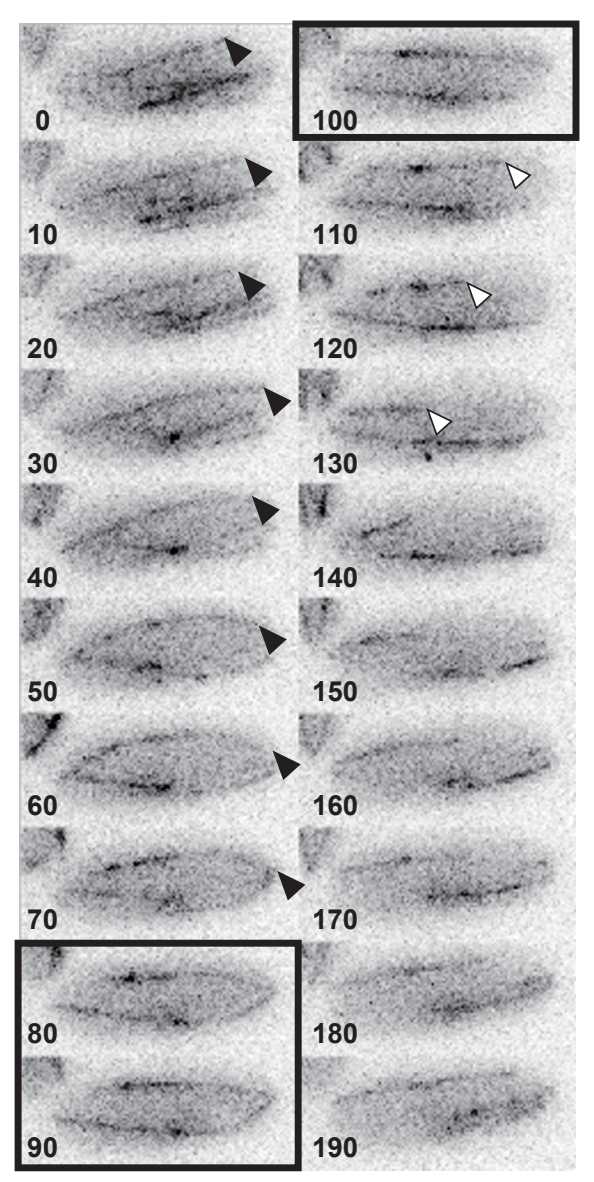

B

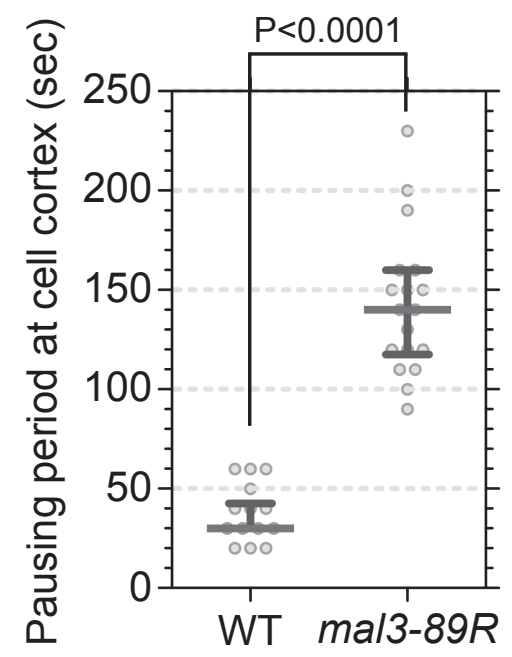

mal3-89R

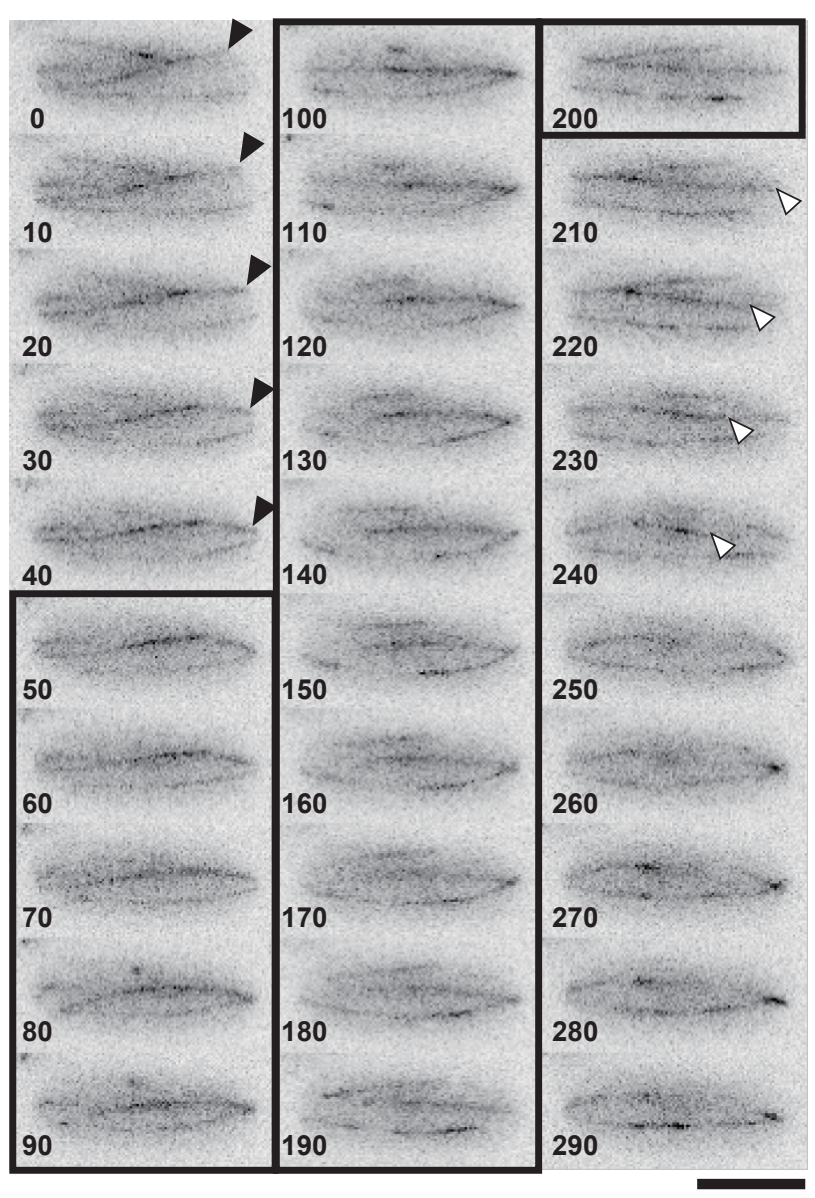

C

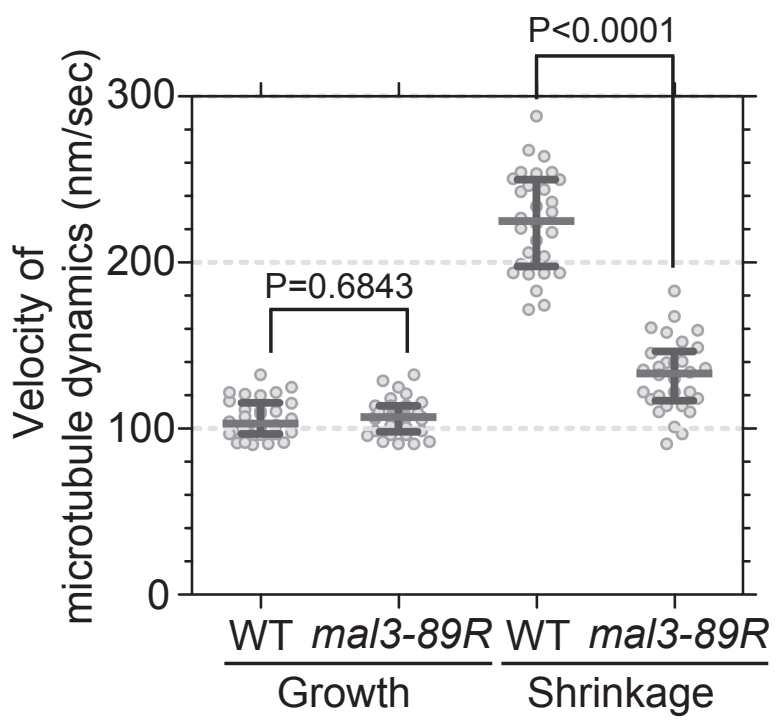




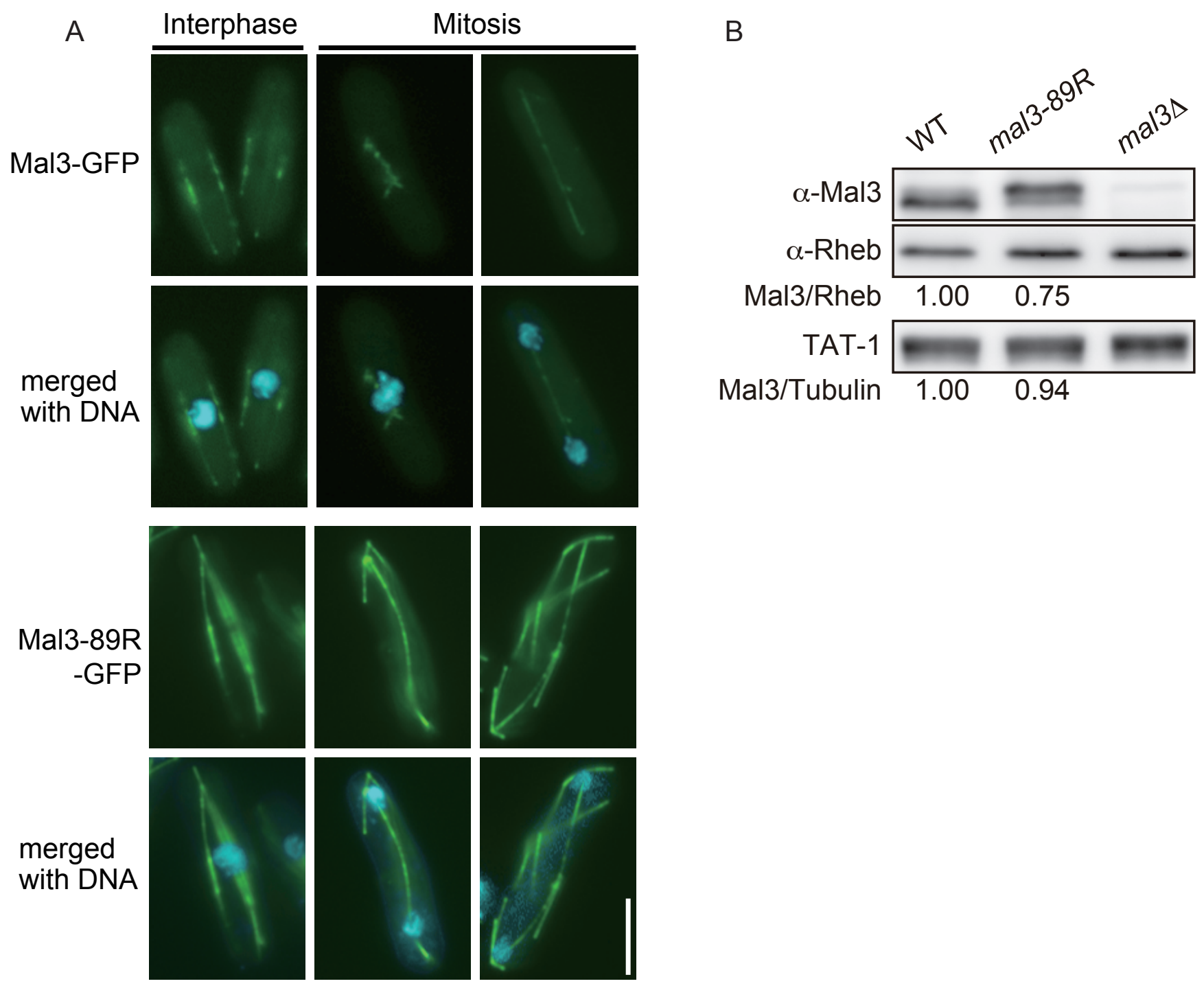

C

WT

mal3-89R
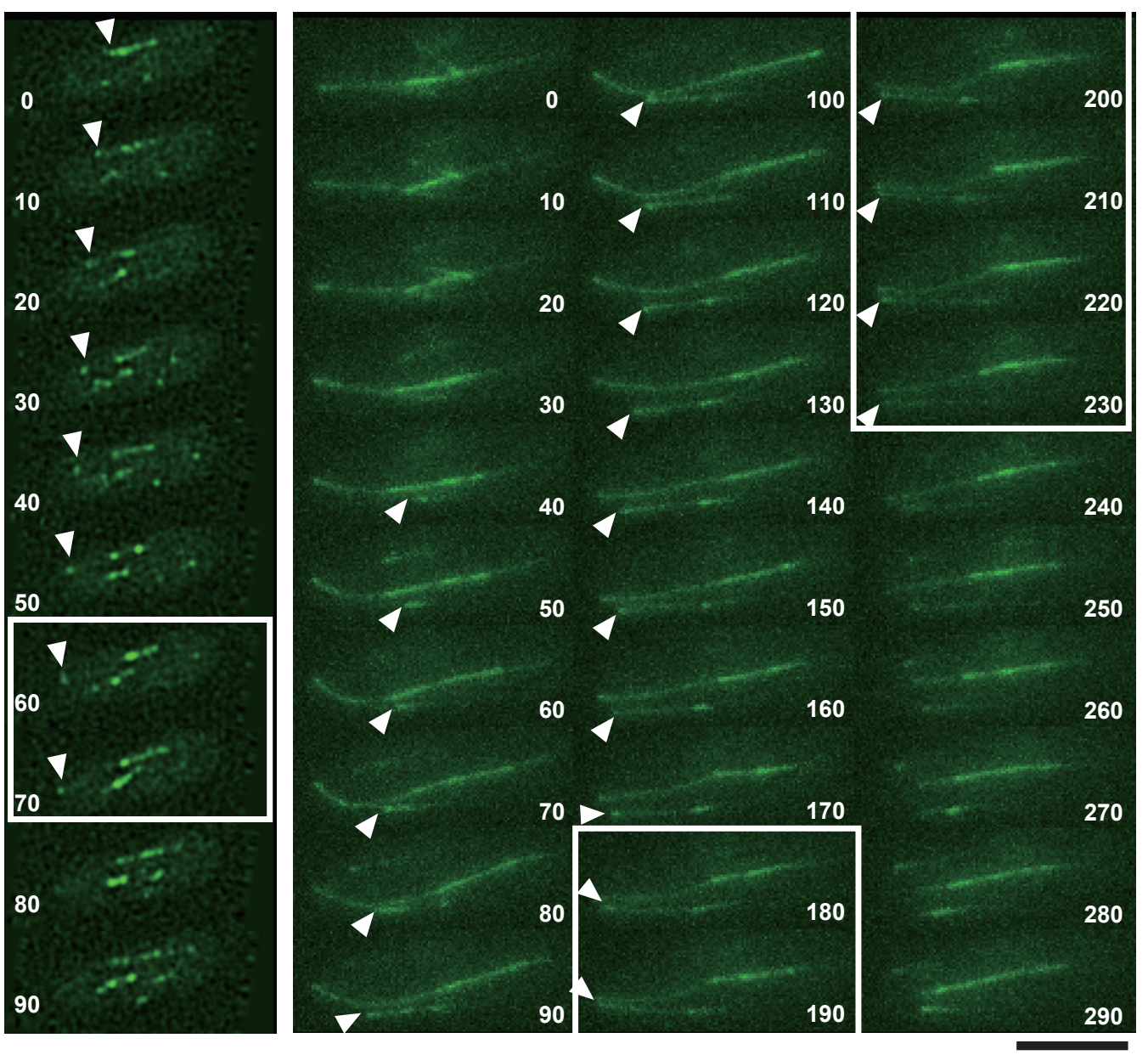

Fig. 4 


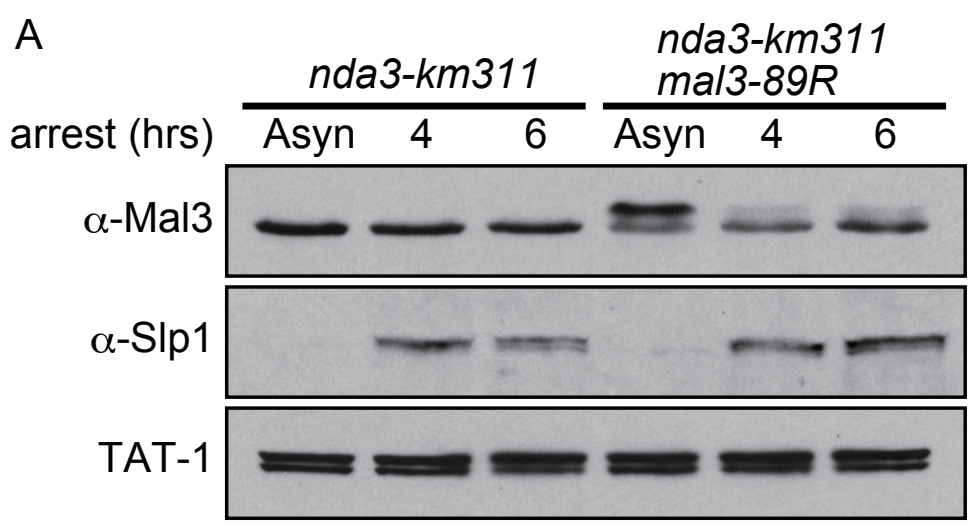

B

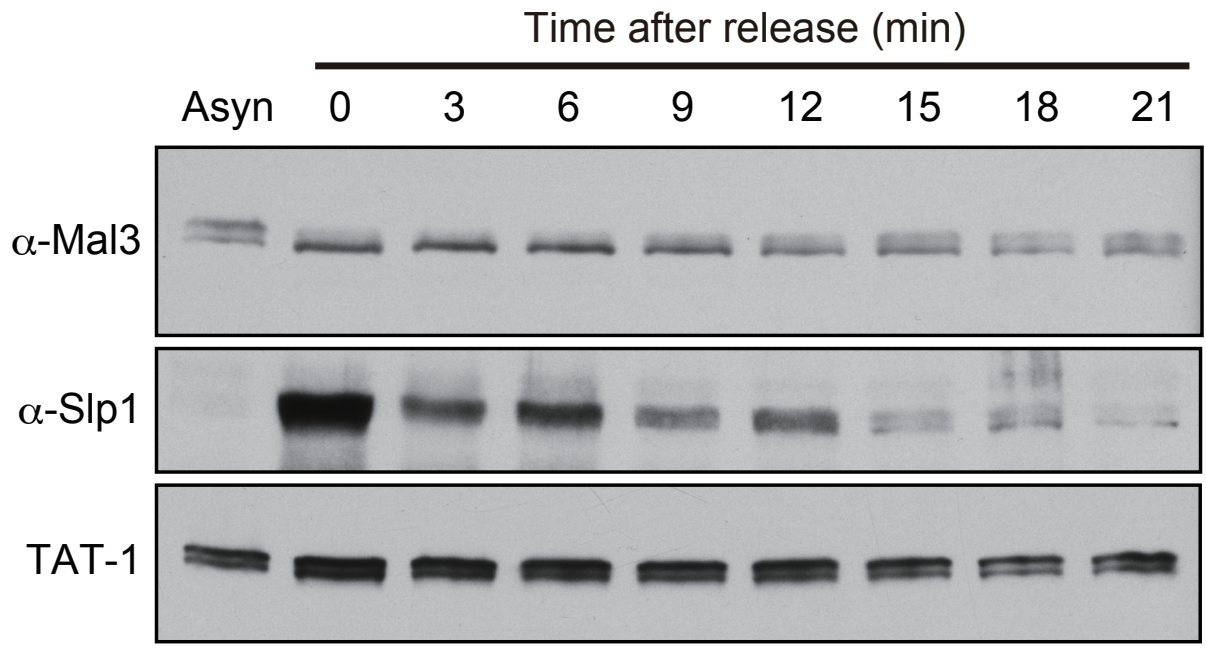

C G1 Time after release $(\mathrm{min})$ arrest $0^{\circ} \mathrm{C} \quad 5 \quad 10 \quad 20$

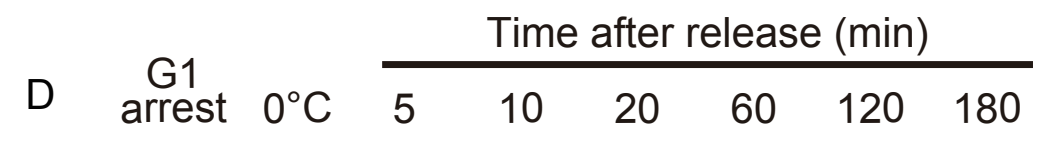

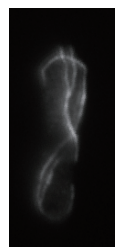

4

1
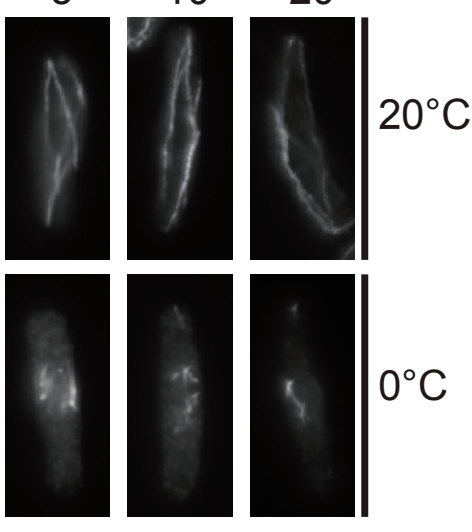

TAT-1

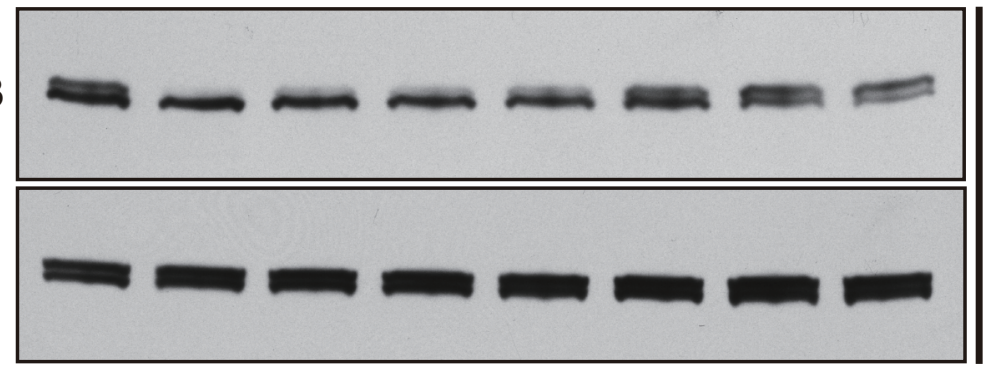

$20^{\circ} \mathrm{C}$

$\alpha-$ Mal3 TAT-1

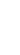

(1)

E

Time after release (hrs)
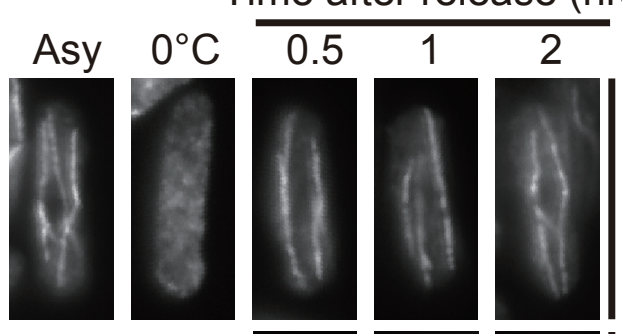

DMSO

F
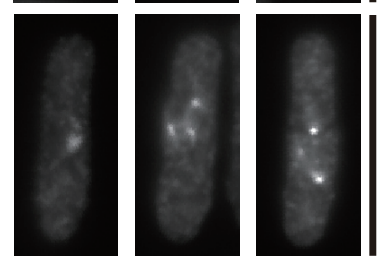

TAT-1

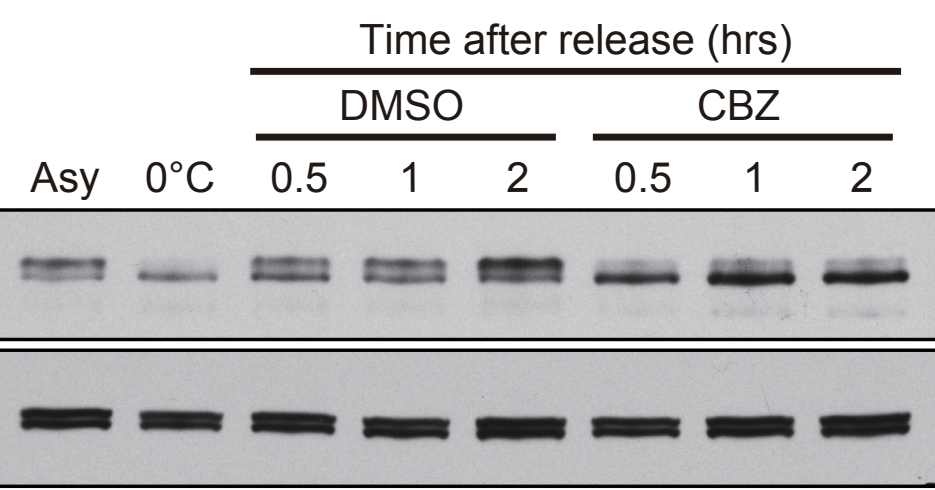

Fig. 5 
A

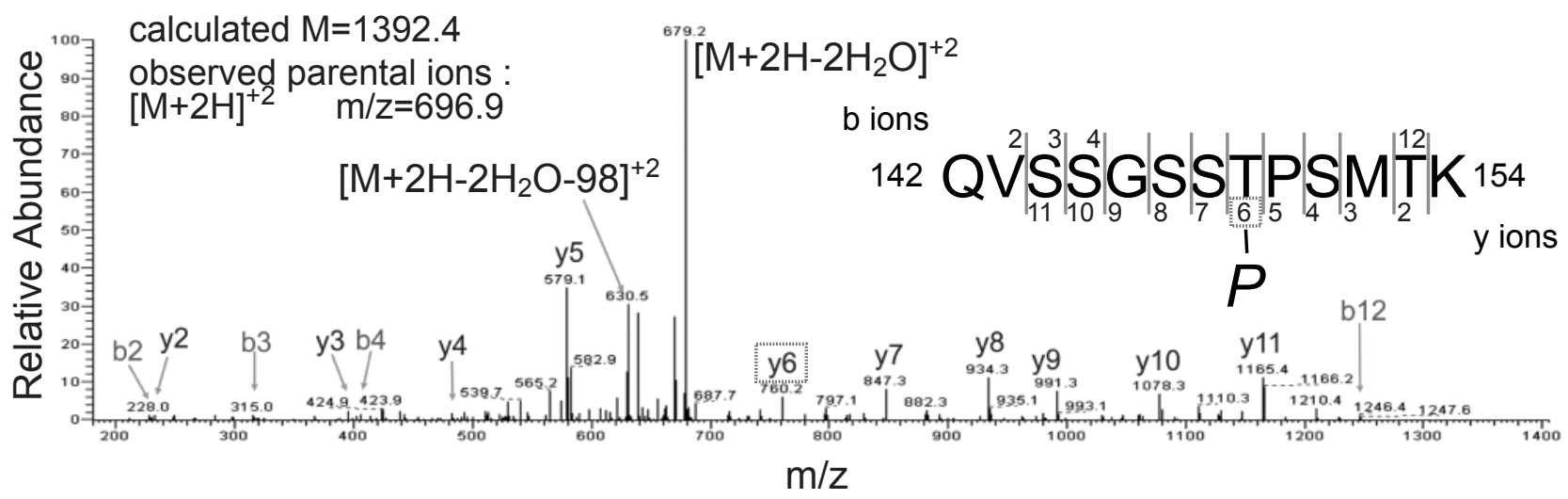

B

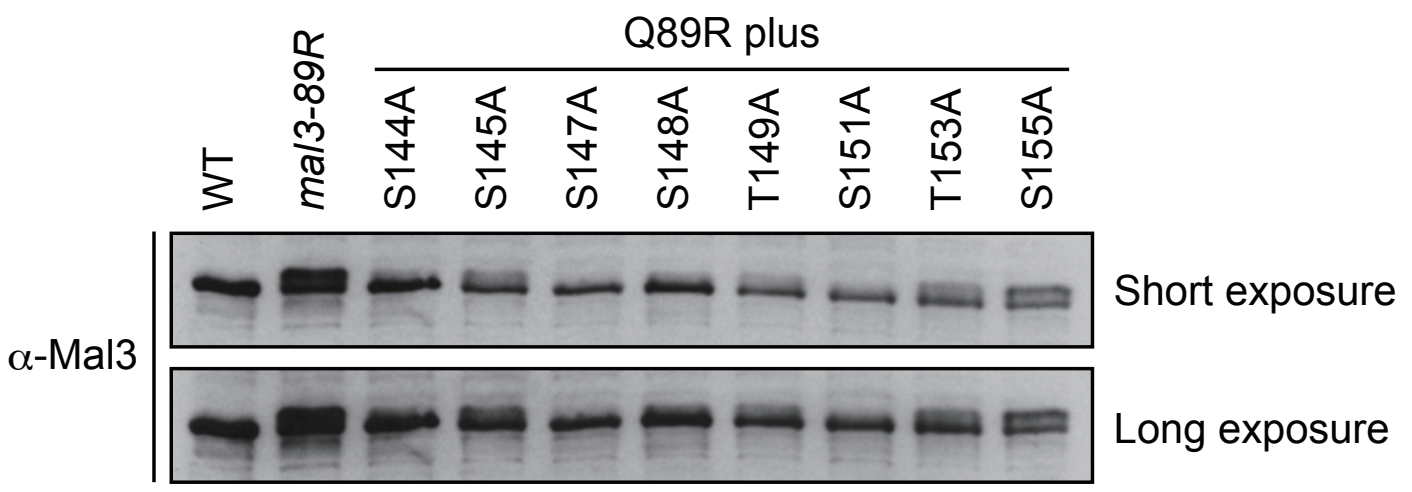

TAT-1

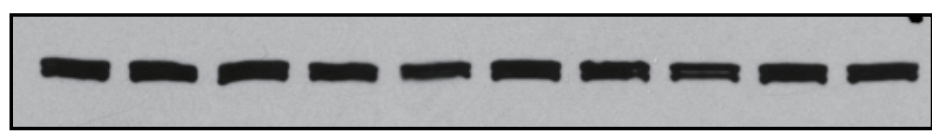

141 RQVSSGSSTPSMTKS 155

C

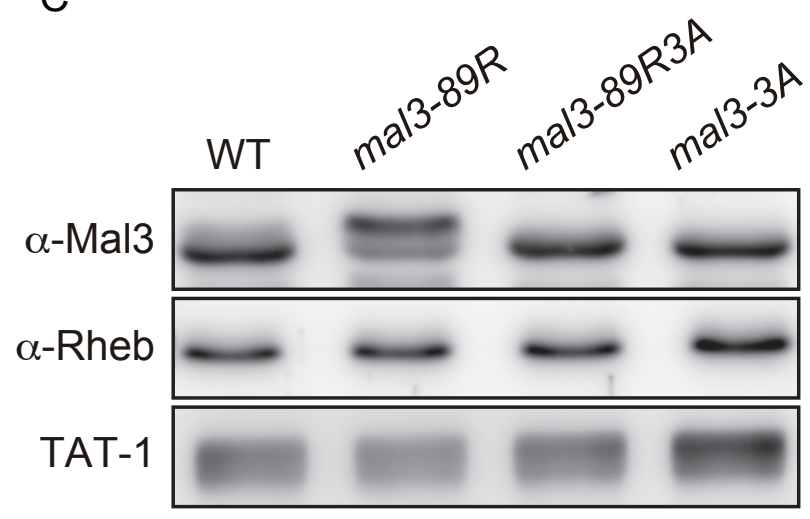

D

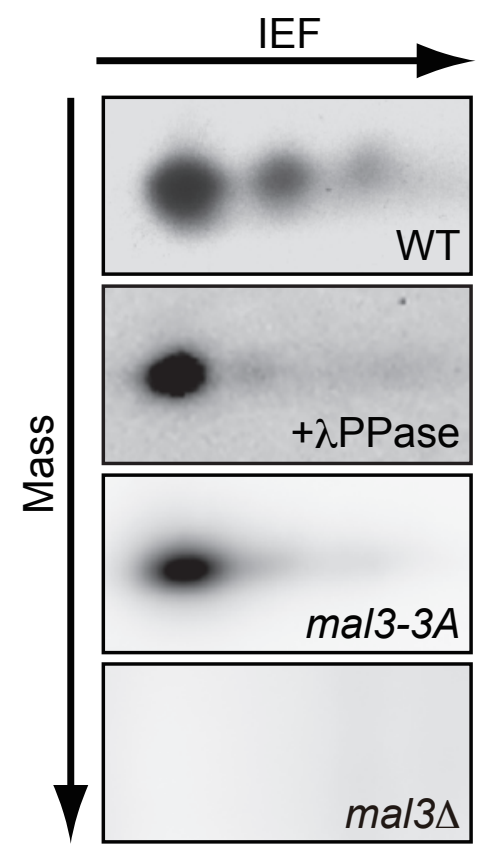

Fig. 6 

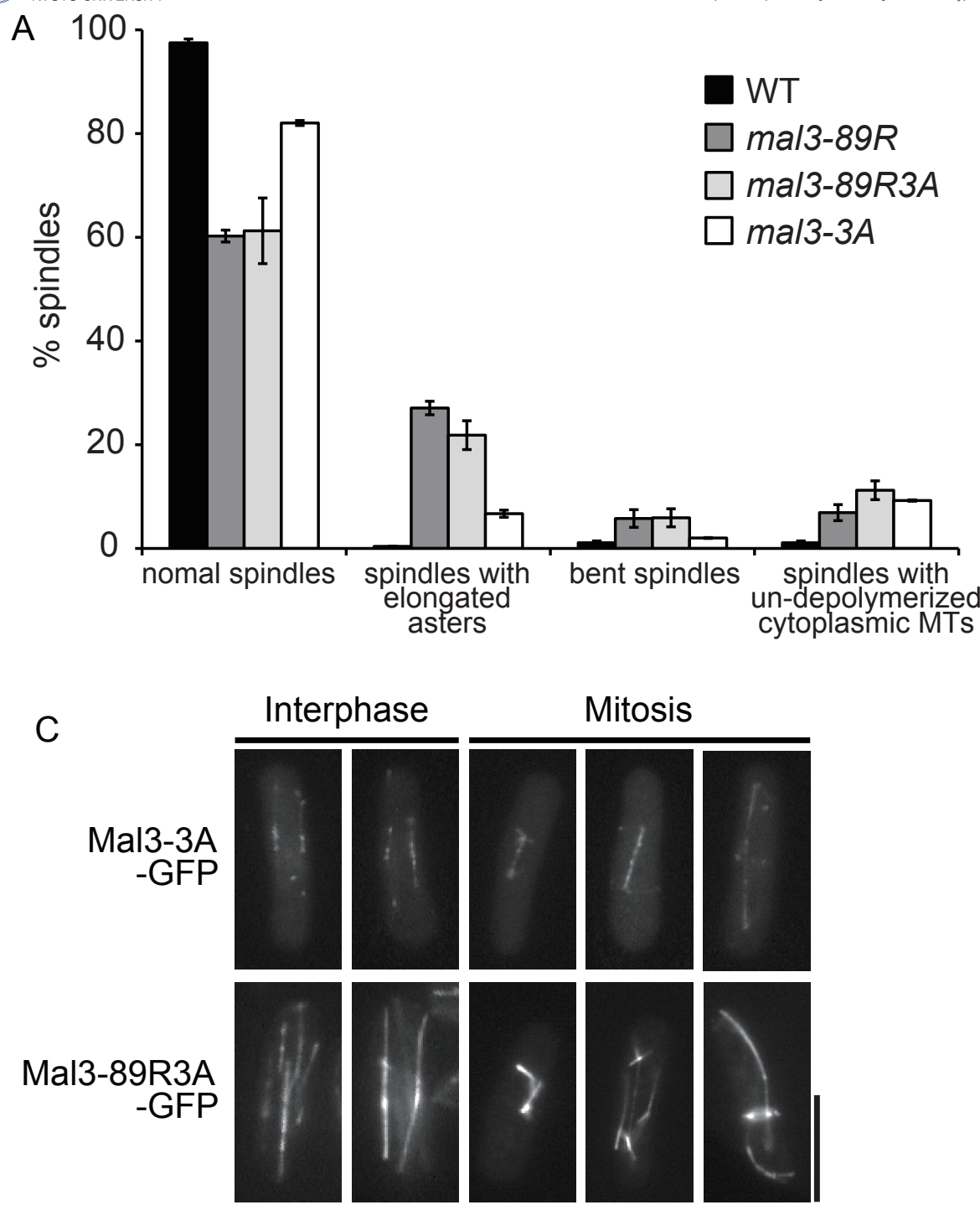

D

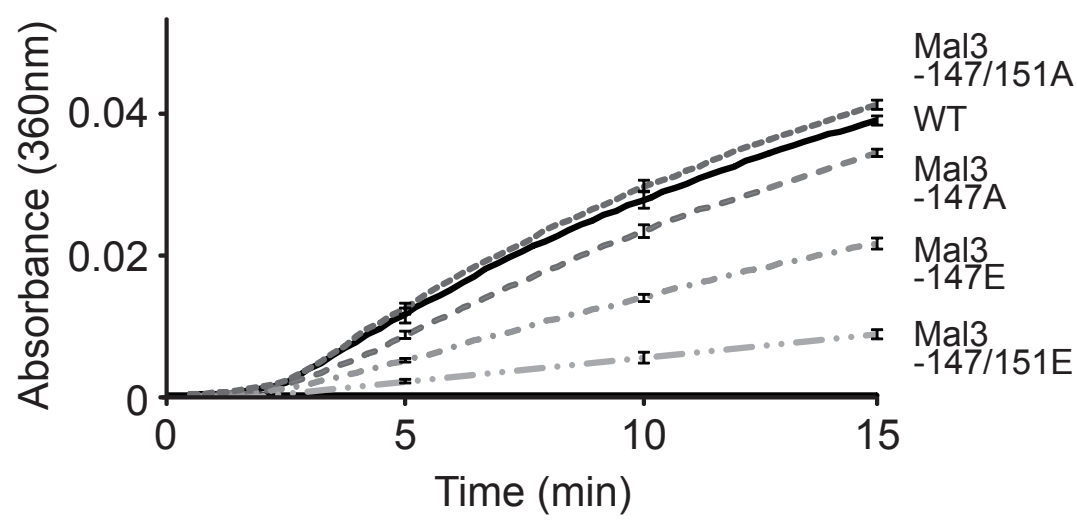

E

Copolymerized MT
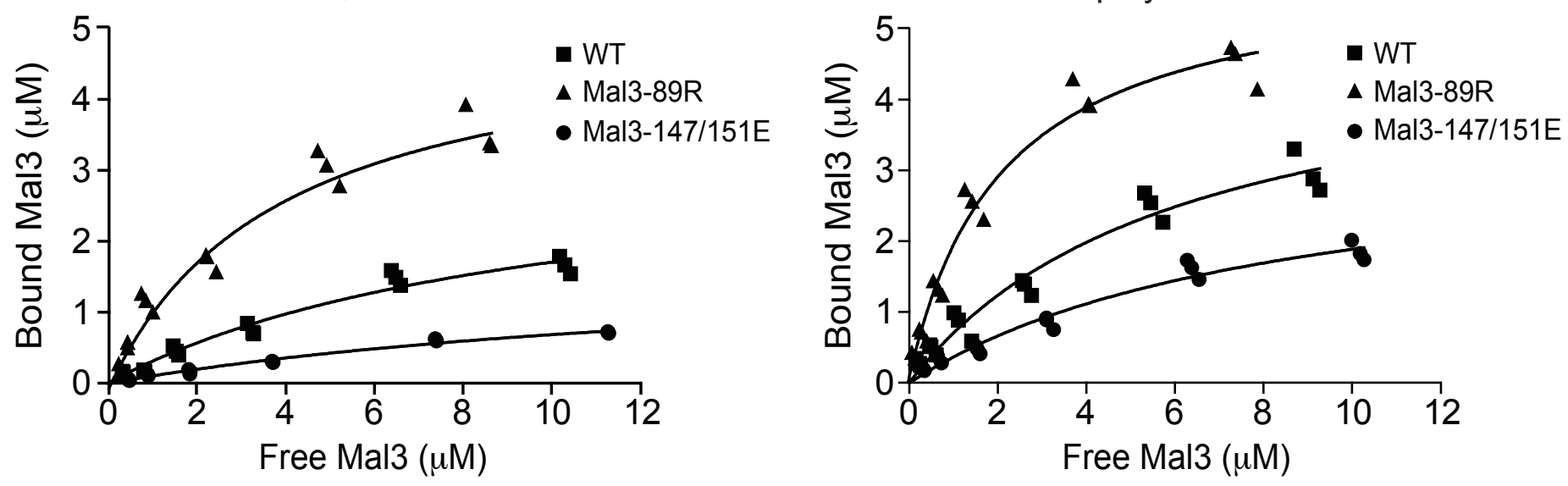
Table 1. mal3-89R mutation affects the stability of non-essential minichromosome

Mini-chromosome loss rate $\left(36^{\circ} \mathrm{C}\right)$

\begin{tabular}{cccc}
\hline & \multicolumn{3}{c}{ Loss rate per cell division (\%) } \\
\cline { 2 - 4 } Strains & 10 & 20 & 40 (generations) \\
\hline WT & 0.54 & 0.58 & 1.15 \\
mal3-89R & 0.42 & 3.60 & 16.15 \\
mal34 & 2.34 & 20.20 & 90.37 \\
\hline
\end{tabular}

Mini-chromosome loss rate $\left(26^{\circ} \mathrm{C}\right)$

\begin{tabular}{cccc}
\hline & \multicolumn{3}{c}{ Loss rate per cell division $(\%)$} \\
\cline { 2 - 4 } Strains & 10 & 20 & 40 \\
\hline WT & 0.41 & 0.96 & 1.07 \\
mal3-89R & 0.53 & 0.32 & 1.71 \\
mal34 & 0.69 & 0.97 & 2.57 \\
\hline
\end{tabular}

Table 2. Binding affinity of Mal3 to microtubule

\begin{tabular}{c|ccc|ccc}
\hline & \multicolumn{3}{|c|}{ Copolymerized MT } & \multicolumn{3}{c}{ Prepolymerized MT } \\
\cline { 2 - 7 } & WT & Mal3-89R & Mal3-147/151E & WT & Mal3-89R & Mal3-147/151E \\
\hline $\mathrm{B}_{\max }(\mu \mathrm{M})$ & $3.4 \pm 0.6$ & $5.2 \pm 0.7$ & $1.8 \pm 0.3$ & $5.0 \pm 0.7$ & $5.9 \pm 0.7$ & $3.5 \pm 0.7$ \\
\hline $\mathrm{K}_{\mathrm{d}}(\mu \mathrm{M})$ & $10.1 \pm 3.0$ & $3.9 \pm 0.9$ & $15.8 \pm 3.5$ & $6.1 \pm 1.7$ & $2.1 \pm 0.4$ & $8.5 \pm 2.6$ \\
\hline
\end{tabular}




\section{Figure Legends}

Fig. S1. Phenotype of the mal3-89R mutant.

(A) Growth defect caused by overexpression of mal3-89R. Wild-type and mal3 deletion mutant strains were transformed with pREP81 (vector), pREP81-mal3 or pREP81-mal3-89R. Each transformant was grown in liquid EMM media containing thiamine, and serial dilutions $\left(5 \times 10^{4}\right.$ to $5 \times 10^{1}$ cells $)$ were spotted on EMM or EMM containing thiamine agar plates. (B) Mitotic arrest caused by overexpression of mal3-89R. The transformants were grown in the absence of thiamine for 16 hours to induce expression of the cloned gene and observed under a fluorescent microscope after staining with DAPI $(\mathrm{a}-\mathrm{f})$. While the transformants with pREP81 or pREP81-mal3 could grow exhibiting normal nuclear morphology ( $a$ and $b$ ), the transformants with pREP81-mal3-89R were arrested with nuclear morphology typical of mitotic defects such as hyper-condensed chromosomes (c), separated and condensed chromosomes (d), “cut"- phenotype (e) and unequally segregated chromosomes (f). Microtubule morphology was also observed in mitosis by immuno-staining with the antibody to $\alpha$-tubulin, TAT-1 in the cells expressing GFP-tagged Sid4 as a marker for SPB, a structure equivalent to centrosome in higher eukaryotes. In the transformants with pREP-81 or pREP81-mal3 (g and h), the spindle microtubules (green) were normally assembled between the two poles (red). In contrast, most of the transformants with pREP81-mal3-89R were arrested with a metaphase-like short spindle with persistent cytoskeltal microtubules, as indicated by arrowhead, (i) or monopolar spindle (j). As shown in the inset of (j), two SPBs slightly separated. The bar indicates $5 \mu \mathrm{m}$. (C) mad2 and bub1 deletion mutant strains were transformed with pREP81 (vector) or 
pREP81-mal3-89R. Each transformant was grown on EMM or EMM containing thiamine agar plates.

Fig. S2. mal3-89R mutant strain was as sensitive as the wild type strain. Wild type, mal3-89R and mal3 deletion mutant strains were grown in liquid YES media, and serial dilutions $\left(5 \times 10^{4}\right.$ to $5 \times 10^{1}$ cells $)$ were spotted on YES agar plates containing $15 \mu \mathrm{g} / \mathrm{ml}$ TBZ. The strains were grown at $32^{\circ} \mathrm{C}$.

Fig. S3. Mitotic defects in mal3-89R. (A and B) Delay in the onset of mitosis. A $c d c 25-22$ mutant and a double mutant, $c d c 25-22$ mal3-89R, both of which were expressing GFP-tagged Sid4 were arrested at the G2/M boundary. The septation index was determined every 10 minutes after the release by counting more than 250 cells (A). In the samples prepared as in (A), the distance between the two poles was determined 20, 30 and 40 minutes after the release by counting more than 100 cells $(B)$. The red lines are the median and the blue bars indicate the inter-quartile range (nonparametric Mann-Whitney $U$ test). (C and D) Time-lapse analysis. A wild-type strain and a mal3-89R mutant, both of which were expressing GFP-tagged Cnp1 (as a marker for centromere) and mCherry-tagged Sad1, were observed by a time-lapse microscope. The images were taken every 2 minutes $(\mathrm{C})$ and the time required for mitosis (defined as time between SPB separation and complete centromere separation) was determined (D). The red lines are the median and the blue bars indicate the inter-quartile range $(n=11$, nonparametric Mann-Whitney $U$ test). 
Fig. S4. Microtubule dynamics in mitosis. The $\alpha$-tubulin was tagged with GFP (GFP-Atb2) in each strain and dynamics of microtubules was observed by live cell imaging. Each panel shows a 10 sec-interval series of maximal projections of the indicated strains. The black arrowheads highlight the tips of aster that will pause for the period indicated by the boxes around the frames. Thus, the astral microtubule in the example of a wild-type cell pauses for $30 \mathrm{sec}$, while in the mal3-89Rmutant, the astral microtubule remains stable for more than $160 \mathrm{sec}$. Numbers in the corner of panels indicate relative timing in seconds.

Fig. S5. Characterization of Mal3-89R protein in mitosis. The wild type and the mutant Mal3 were tagged with GFP and examined by live cell imaging. Each panel shows a 10-sec-interval series of maximal projections of the indicated strains. The white arrowheads highlight the tips of aster that will pause for the period indicated by the boxes around the frames. The red arrowheads indicate a remaining cytoplasmic microtubule in mitosis and the yellow arrowheads the SPBs. The bar indicates $5 \mu \mathrm{m}$.

Fig. S6. Phosphatase assay of Mal3-89R protein. The Mal3-89R proteins purified by immunoprecipitation with the antibody to Mal3 were treated with or without 100 units of lambda phosphatase (New England Biolabs), and 1mM sodium orthovanadate was added as a phosphatase inhibitor. The reaction solution was incubated for 30 minutes at $30^{\circ} \mathrm{C}$. The asterisk indicates a non-specific band.

Fig. S7. Microtubule binding assay. (A) Recombinant protein of Mal3 mutants with an $\mathrm{N}$-terminal His-tag was expressed in BL21 (DE3) and purified with Ni-NTA Superflow 
Cartride (Qiagen) and HiTrapQ (GE Healthcare). (B) Typical gel run of bovine brain tubulin, presence of GTP with or without taxol, and recombinant protein of Mal3 mutants for microtubule binding assay. (C) Effect on microtubule binding assay. Wild type Mal3, Mal3-89R and phospho-mimetic mutant proteins (added concentration, 0.5-12 $\mu \mathrm{M}$, are indicated) were measured the binding affinity for copolumerized (upper) or prepolumerized (taxol-stabilized; lower) microtubules. S, supernatant fraction; P, pellet fraction. 
A

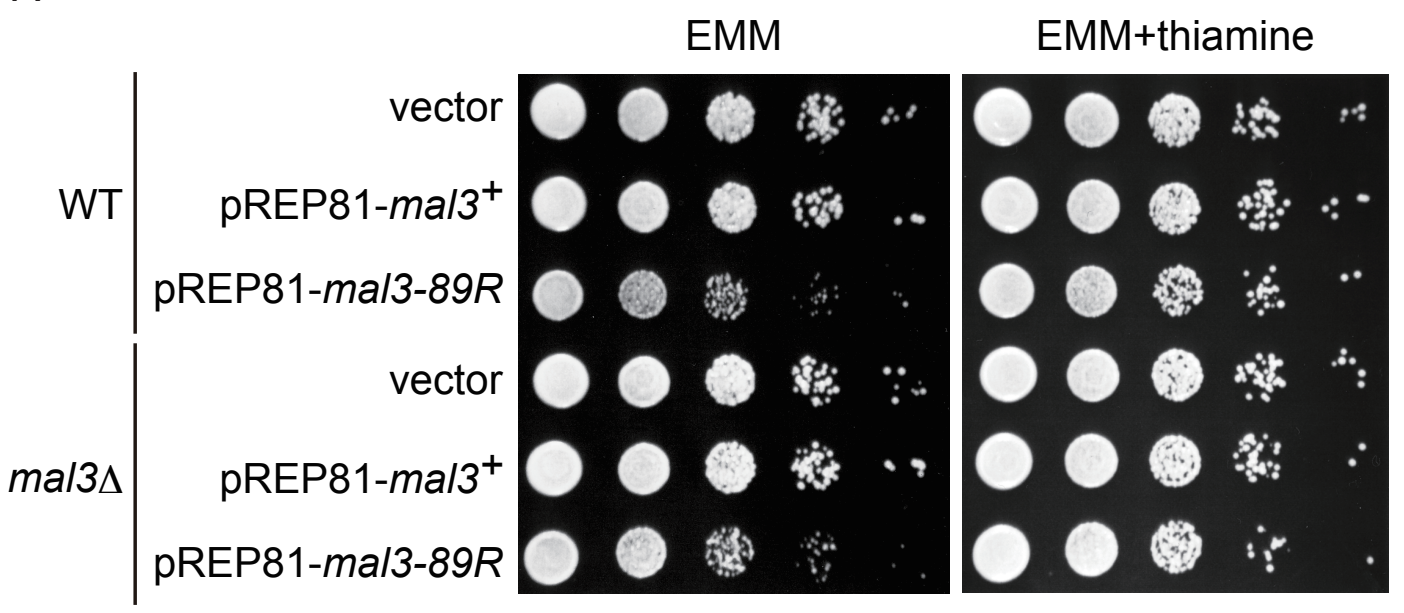

B
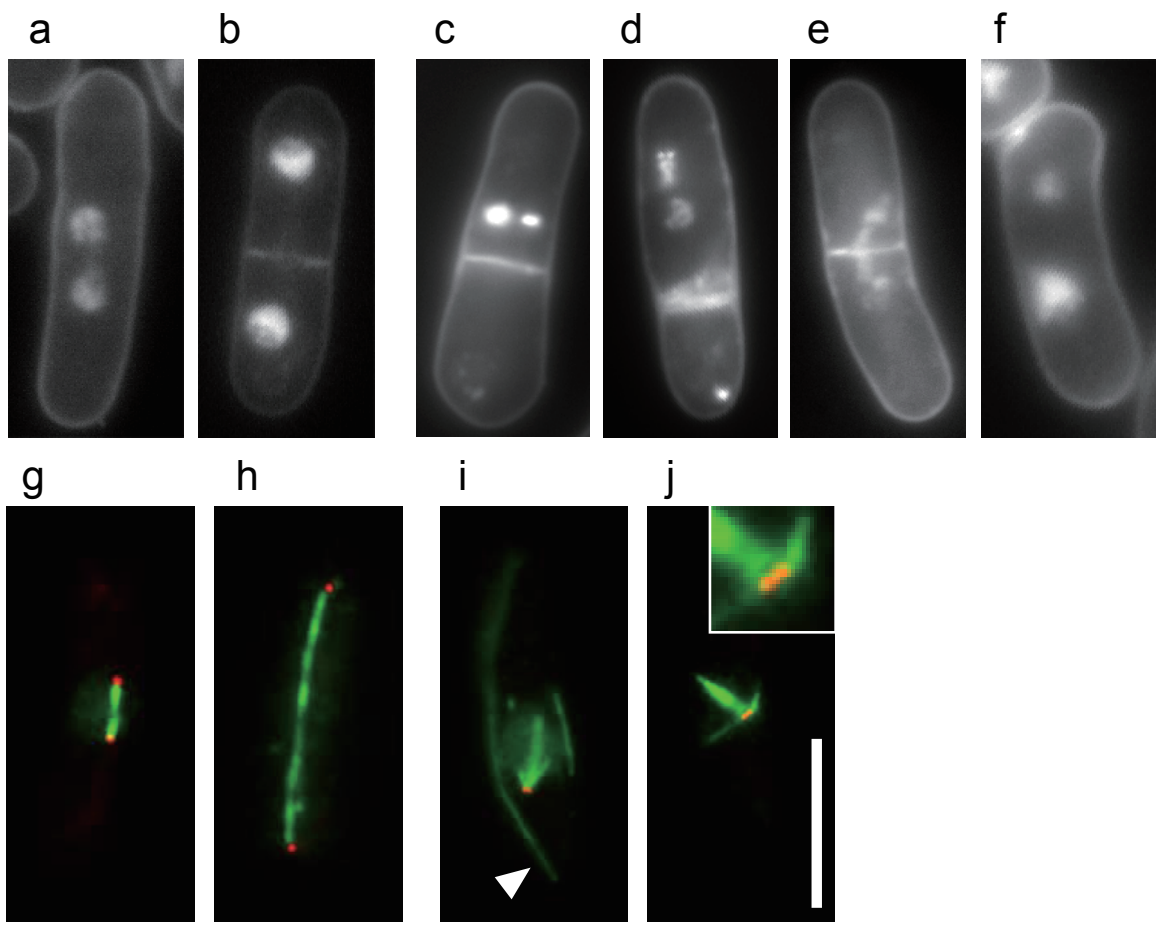

C
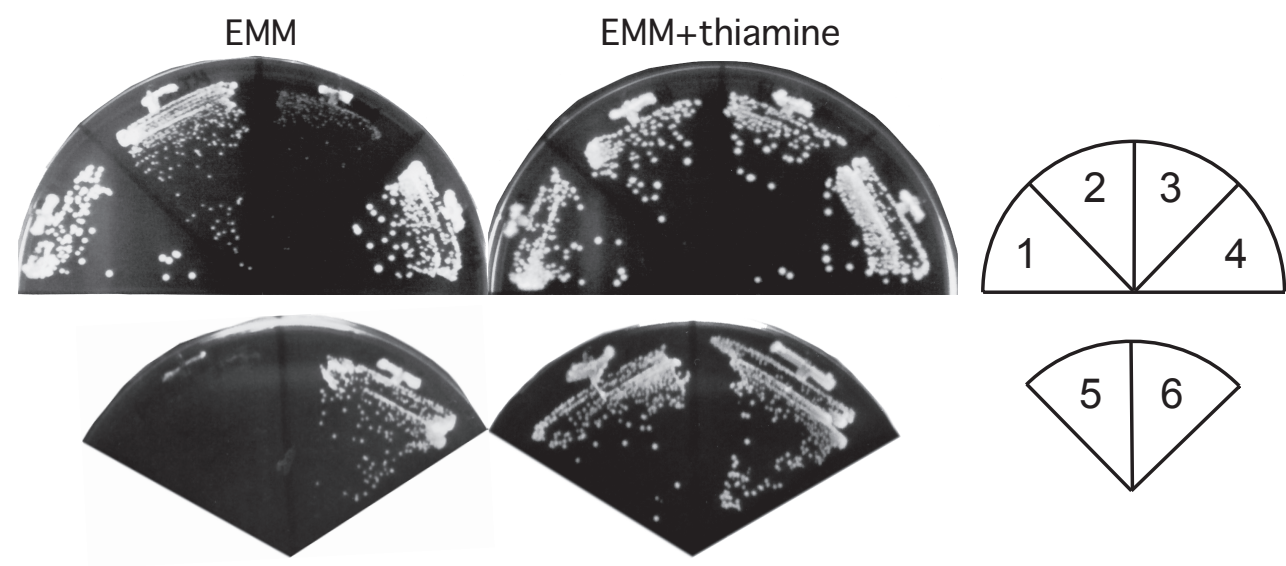

1: WT/ vector

2: WT/ pREP81-mal3-89R

3: $\operatorname{mad} 2 \Delta / \mathrm{pREP} 81-\mathrm{mal3}-89 R$

4: $\operatorname{mad} 2 \Delta /$ vector

5: bub1 $/$ pREP81-mal3-89R

6: bub1 $1 \Delta$ vector

Fig. S1 


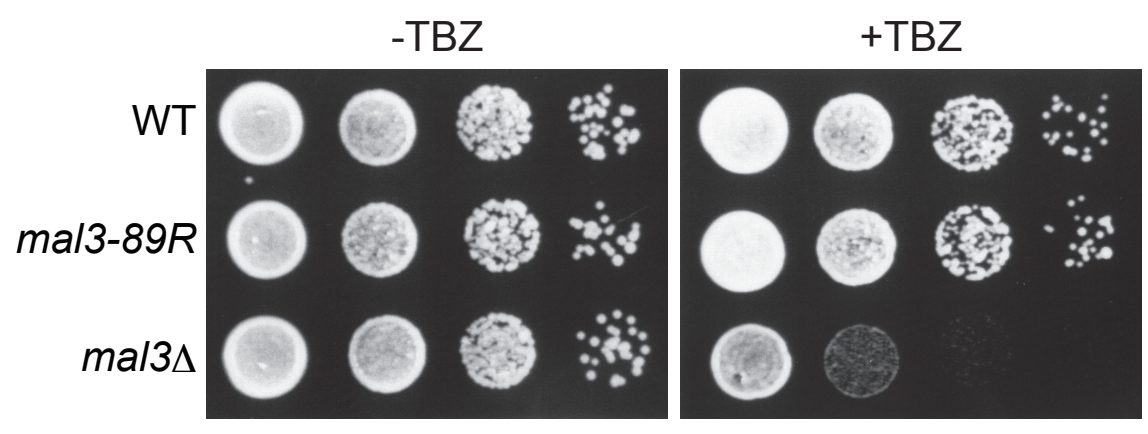

Fig.S2 
A

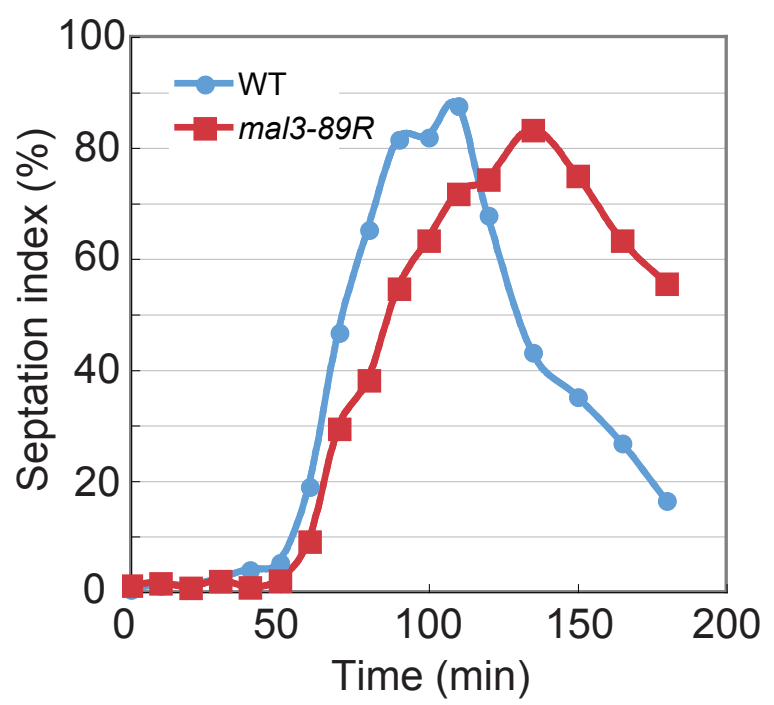

C

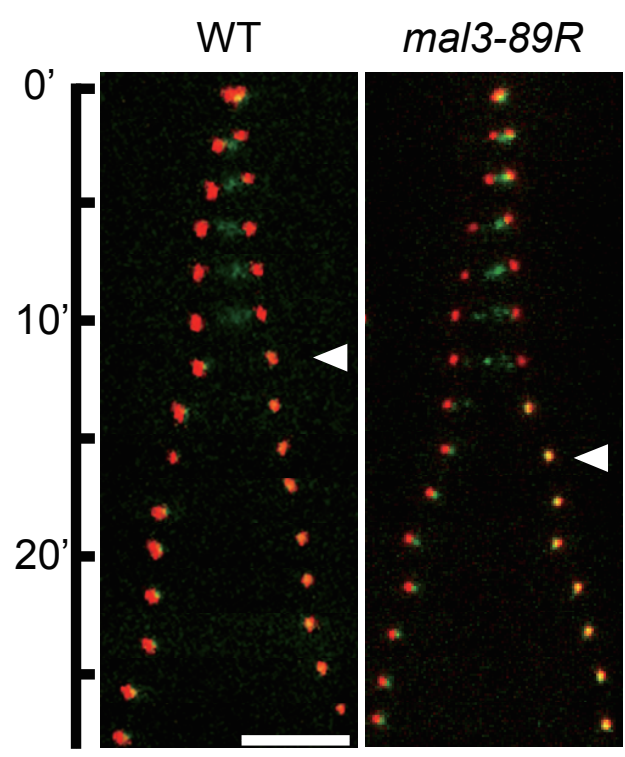

B
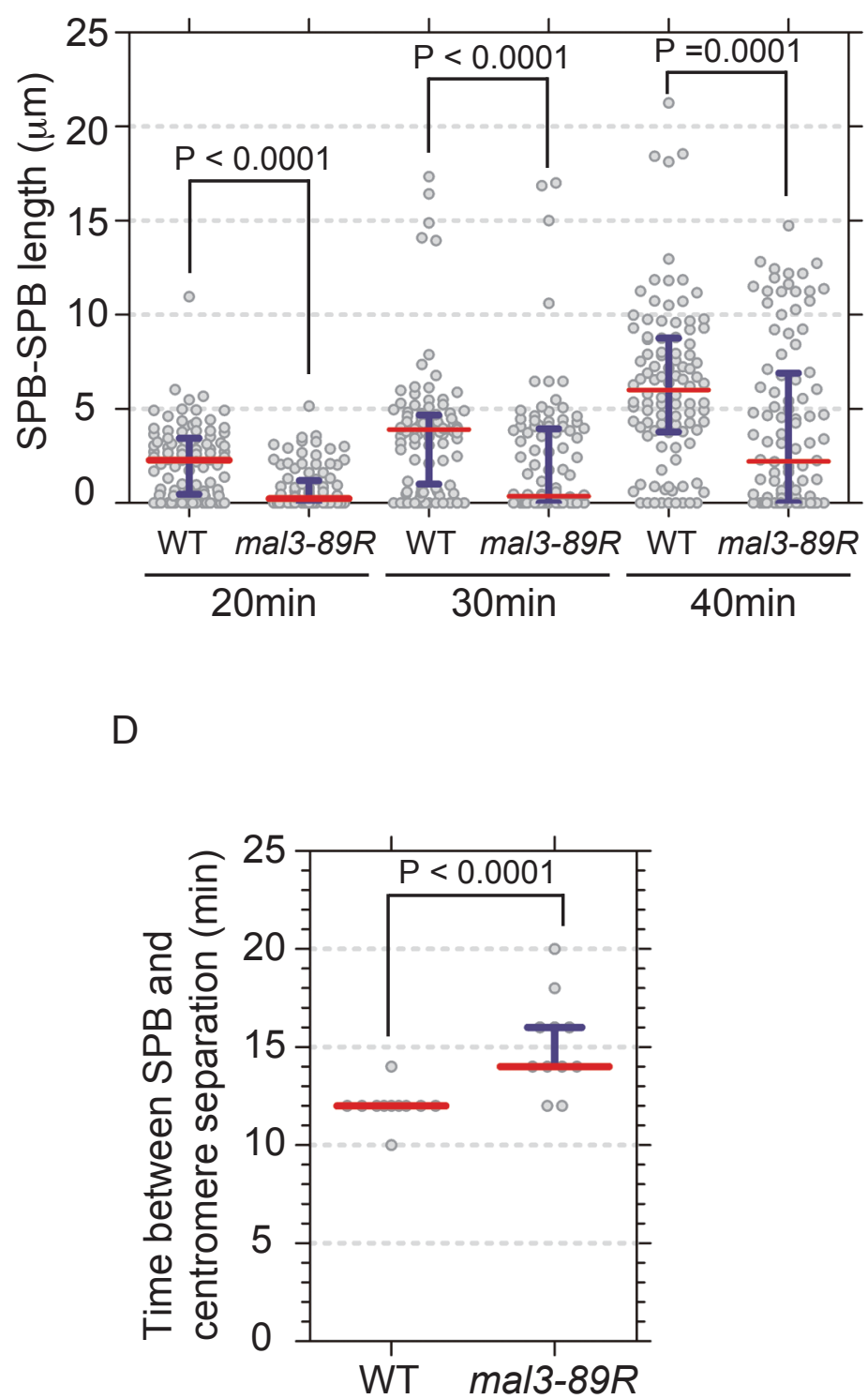

Fig. S3 
WT

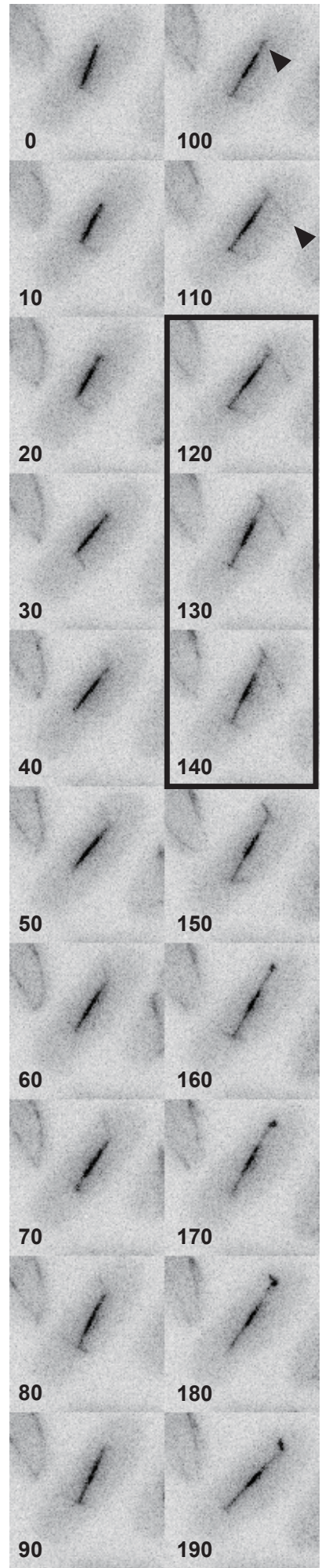

mal3-89R

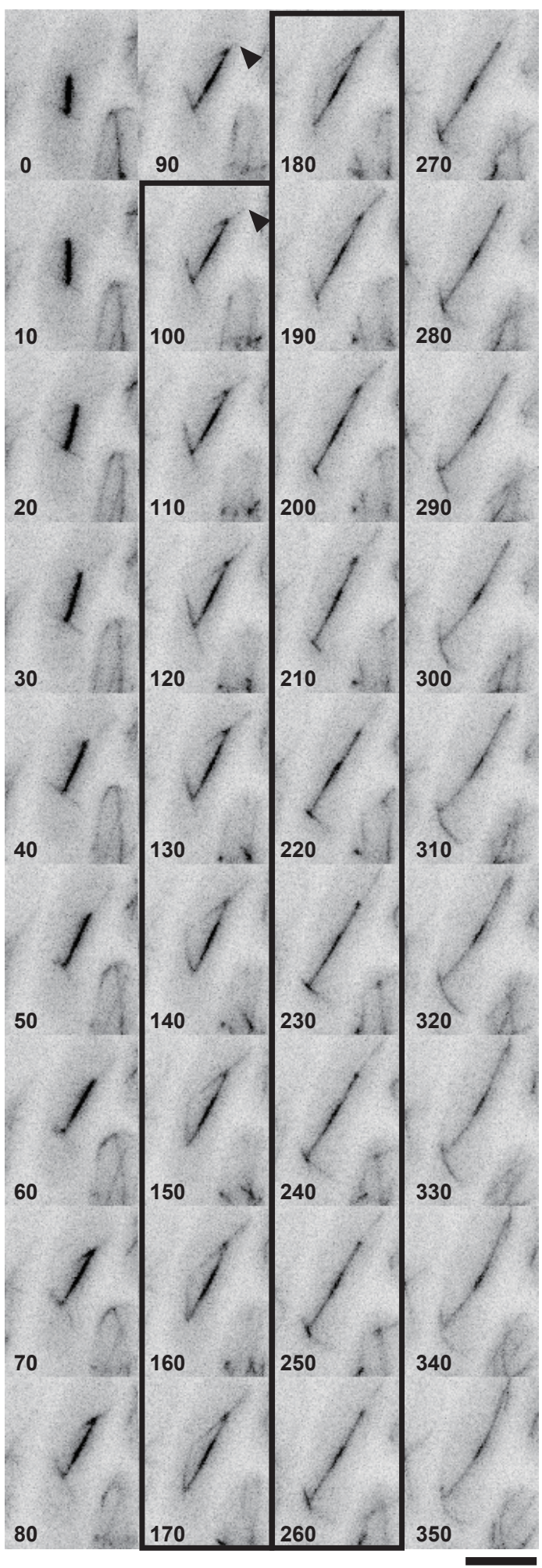

Fig.S4 
WT

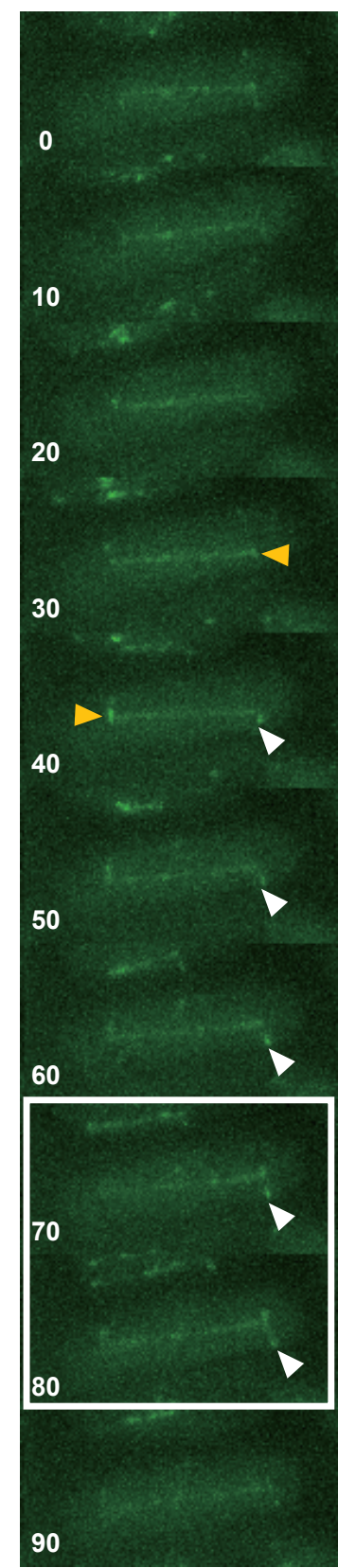

10
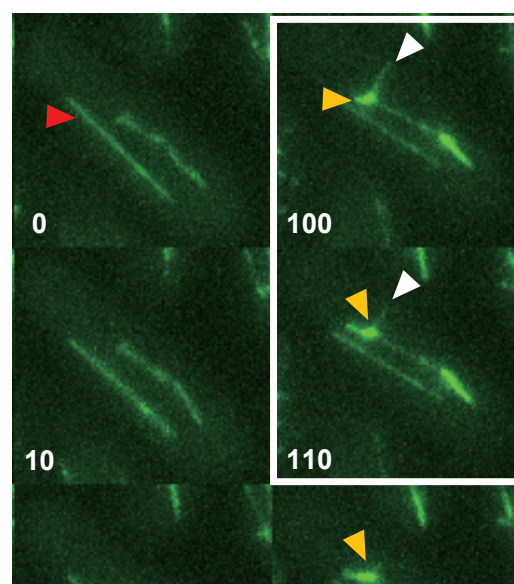

20

120

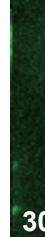

30

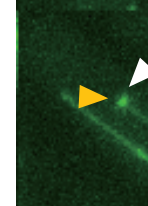

40

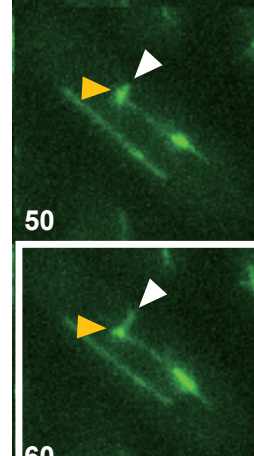

50

60

160

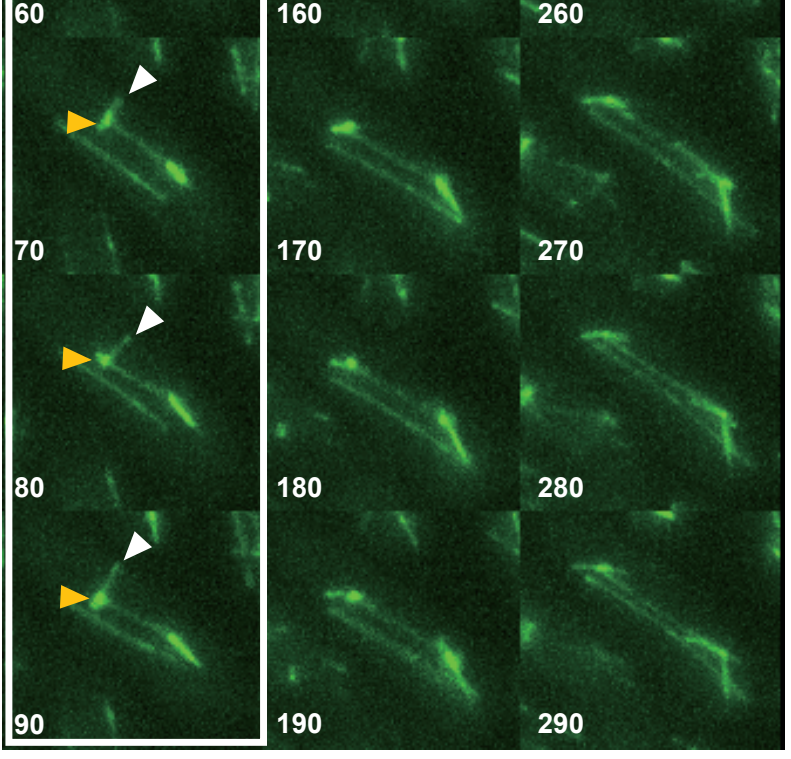

200

220

230

250
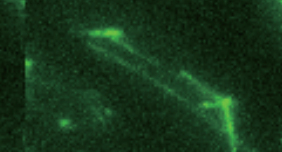

260

240

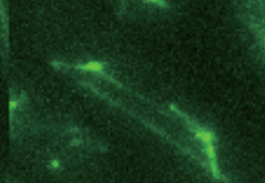

$$
\text { . }
$$

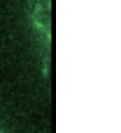




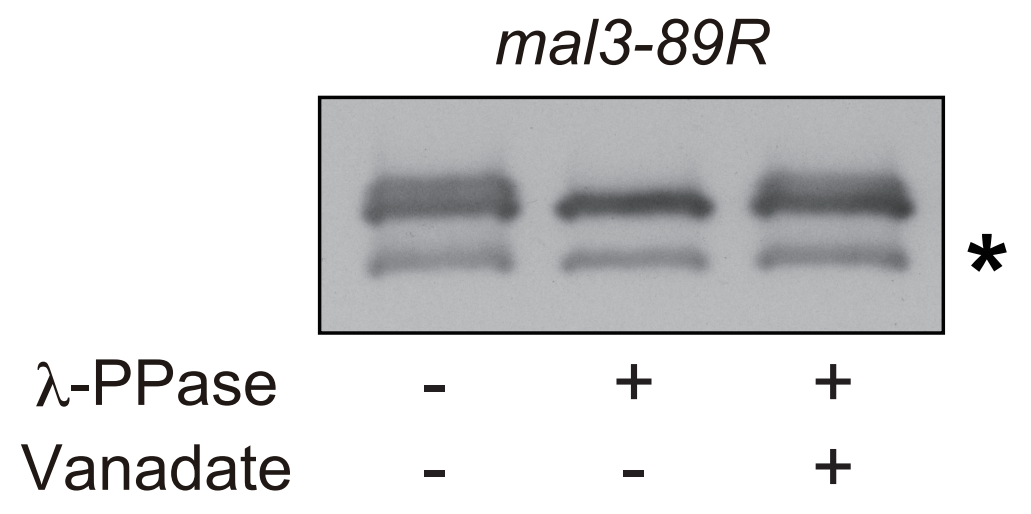

Fig.S6 
A

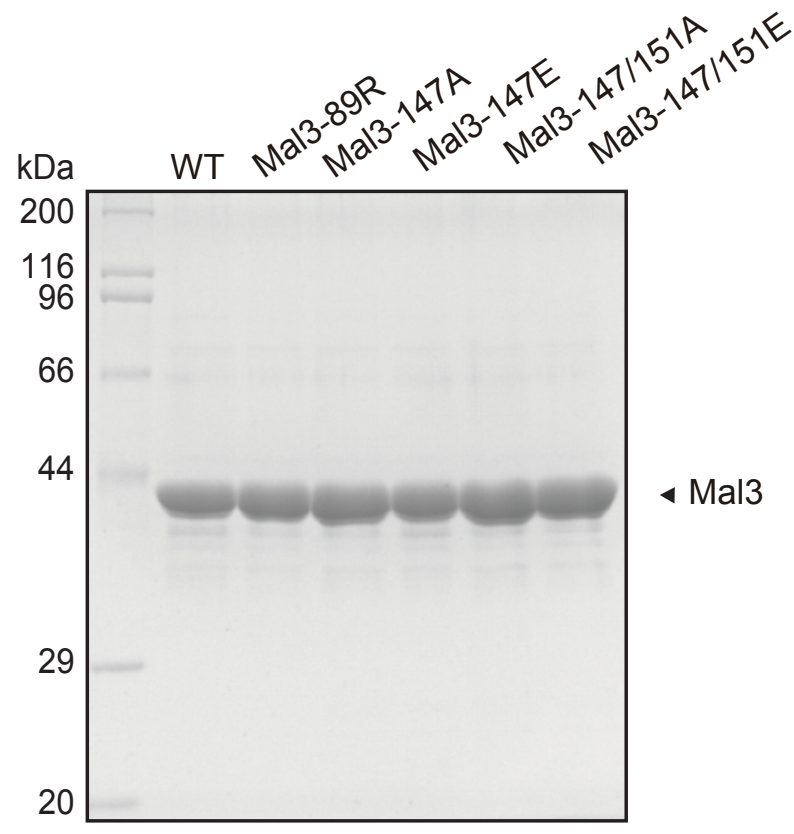

B

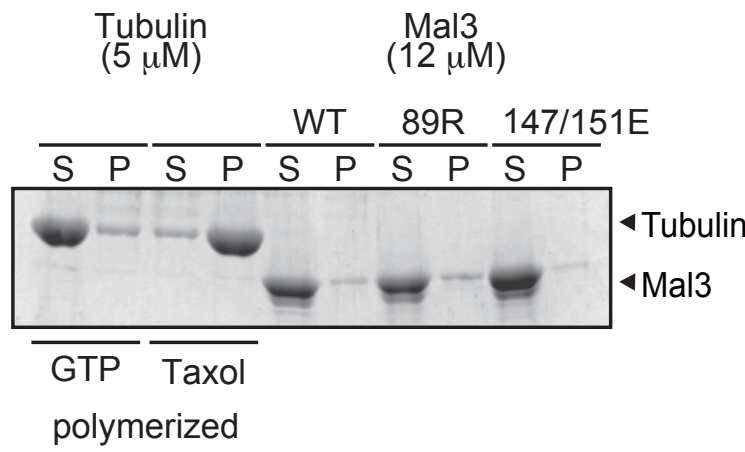

C

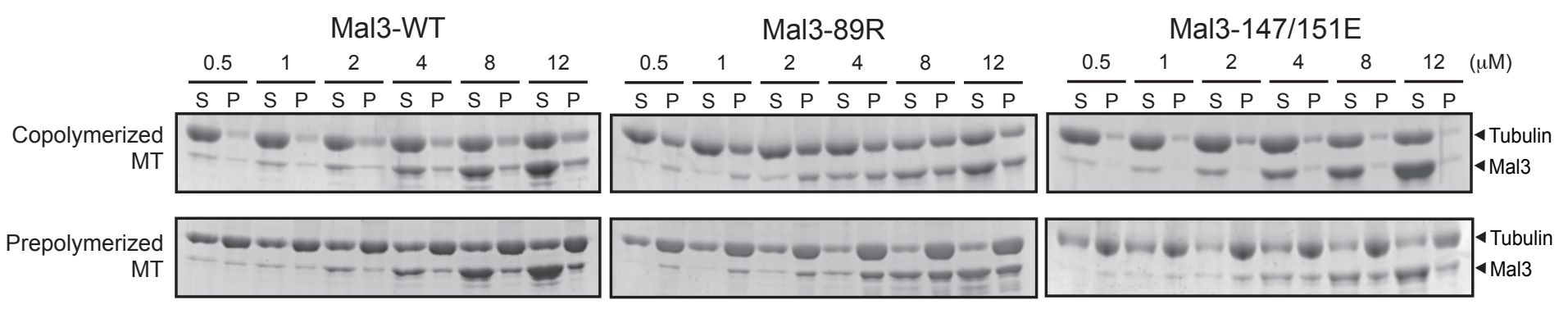

Fig.S7 


\section{Supplementary table. Yeast strains used in this s}

\begin{tabular}{|c|c|c|}
\hline Strain & Genotype & Source \\
\hline 972 & $h^{-}$ & Lab stock \\
\hline SP 6 & $h^{-}$leu1-32 & Lab stock \\
\hline RBC 149 & $h^{-}$mal3::ura4 ${ }^{+}$ura4-D18 & This study \\
\hline RBC 150 & $h^{-}$mal3::ura4 ${ }^{+}$leu1-32 ura4-D18 & This study \\
\hline RBC 151 & $h^{-}$pREP81:LEU2 leu1-32 & This study \\
\hline RBC 152 & $h^{-}$mal3-pREP81:LEU2 leu1-32 & This study \\
\hline RBC 153 & $h^{-}$mal3-GFP:LEU2 leu1-32 & This study \\
\hline RBC 154 & $h^{-}$mal3::ura4 ${ }^{+}$pREP81:LEU2 leu1-32 ura4-D18 & This study \\
\hline RBC 155 & $h^{-}$mal3::ura4 ${ }^{+}$mal3-pREP81:LEU2 leu1-32 ura4-D18 & This study \\
\hline RBC 156 & $h^{-}$ade6-M210 leu1-32 ch $^{16}[$ ade6-M216] & This study \\
\hline RBC 157 & $h^{-}$mal3::ura4 ${ }^{+}$ade6-M210 leu1-32 ura4-D18 ch $^{16}[$ ade6-M216] & This study \\
\hline RBC 158 & $h^{+}$nda3-km311 mad2-GFP:LEU2 leu1-32 & This study \\
\hline RBC 159 & $h^{-}$cdc25-22 sid4-GFP:kan ${ }^{R}$ leu1-32 & This study \\
\hline RBC 160 & $h^{+}$sad1-mCherry:kan ${ }^{R}$ GFP-cnp1:hph leu1-32 & This study \\
\hline RBC 161 & $h^{-}$mal3-89R leu1-32 & This study \\
\hline RBC 162 & $h^{+}$lys1:Pnda3-GFP-atb2 leu1-32 & This study \\
\hline RBC 163 & $h^{-}$mal3::ura4 ${ }^{+}$lys1:Pnda3-GFP-atb2 leu1-32 ura4-D18 & This study \\
\hline RBC 164 & $h^{-}$leu1-32 mal3-89R-pREP81:LEU2 & This study \\
\hline RBC 165 & $h^{-}$bub1::ura4 ${ }^{+}$pREP81:LEU2 ade6-210 leu1-32 ura4-D18 & This study \\
\hline RBC 166 & $h^{-}$bub1::ura4 ${ }^{+}$mal3-89R-pREP81:LEU2 ade6-210 leu1-32 ura4-D18 & This study \\
\hline RBC 167 & $h^{-}$mad2::ura4 ${ }^{+}$pREP81:LEU2 leu1-32 ura4-D18 & This study \\
\hline RBC 168 & $h^{-}$mad2::ura4 ${ }^{+}$mal3-89R-pREP81:LEU2 leu1-32 ura4-D18 & This study \\
\hline RBC 169 & $h^{-}$mal3-89R ade6-M210 leu1-32 ch $^{16}[$ ade6-M216] & This study \\
\hline RBC 170 & $h^{-}$mal3-89R-GFP:LEU2 leu1-32 & This study \\
\hline RBC 171 & $h^{-} \mathrm{mal3}-89 R$ & This study \\
\hline RBC 172 & $h^{+}$mal3-89R nda3-km311 mad2-GFP:LEU2 leu1-32 & This study \\
\hline RBC 173 & h- mal3-89R cdc25-22 sid4-GFP:kanR leu1-32 & This study \\
\hline RBC 174 & $h^{+}$mal3-89R sad1-mCherry:kan ${ }^{R}$ GFP-cnp1:hph leu1-32 & This study \\
\hline RBC 175 & $h^{+}$mal3-89R cdc10-M17 leu1-32 & This study \\
\hline RBC 176 & $h^{+}$mal3-147E sad1-mCherry:kan ${ }^{R}$ GFP-cnp1:hph leu1-32 & This study \\
\hline RBC 177 & $h^{+}$mal3-147/151E sad1-mCherry:kan ${ }^{R}$ GFP-cnp1:hph leu1-32 & This study \\
\hline RBC 178 & $h^{+}$mal3-89R lys1:Pnda3-GFP-atb2 leu1-32 & This study \\
\hline RBC 179 & h mal3-89R3A lys1:Pnda3-GFP-atb2 leu1-32 & This study \\
\hline
\end{tabular}

